\title{
SEMILINEAR STOCHASTIC EQUATIONS IN A HILBERT SPACE WITH A FRACTIONAL BROWNIAN MOTION*
}

\author{
T. E. DUNCAN ${ }^{\dagger}$, B. MASLOWSKI ${ }^{\ddagger}$, AND B. PASIK-DUNCAN $^{\dagger}$
}

\begin{abstract}
The solutions of a family of semilinear stochastic equations in a Hilbert space with a fractional Brownian motion are investigated. The nonlinear term in these equations has primarily only a growth condition assumption. An arbitrary member of the family of fractional Brownian motions can be used in these equations. Existence and uniqueness for both weak and mild solutions are obtained for some of these semilinear equations. The weak solutions are obtained by a measure transformation that verifies absolute continuity with respect to the measure for the solution of the associated linear equation. Some examples of stochastic differential and partial differential equations are given that satisfy the assumptions for the solutions of the semilinear equations.
\end{abstract}

Key words. semilinear stochastic equations, fractional Brownian motion, stochastic partial differential equations, absolute continuity of measures

AMS subject classifications. 60H15, 60G18, 60G15

DOI. $10.1137 / 08071764 \mathrm{X}$

1. Introduction. Fractional Brownian motion denotes a family of Gaussian processes with continuous sample paths that are indexed by the Hurst parameter $H \in(0,1)$ and that have properties that appear empirically in a wide variety of physical phenomena, such as hydrology, economic data, telecommunications, and medicine. Since some physical phenomena are naturally modeled by stochastic partial differential equations and the randomness can be described by a fractional Gaussian noise, it is important to study the problems of the solutions of stochastic differential equations in a Hilbert space with a fractional Brownian motion. A significant family of these stochastic equations is the set of semilinear equations, so it is important to investigate the existence and the uniqueness of the solutions of the equations and the sample path properties of the solutions. If primarily only some growth assumptions are made on the nonlinear terms in the semilinear equations, then it is natural to investigate weak solutions, especially those that arise by an absolutely continuous transformation of the measure of the solution of the associated linear stochastic equation.

The study of the solutions of stochastic equations in an infinite-dimensional space with a (cylindrical) fractional Brownian motion (for example, stochastic partial differential equations) has been relatively limited. For the Hurst parameter $H \in(1 / 2,1)$, linear and semilinear equations with an additive fractional Gaussian noise, the formal derivative of a fractional Brownian motion, are considered in $[8,13,15,28]$. Random dynamical systems described by such stochastic equations and their fixed points are studied in [22]. A pathwise (or nonprobabilistic) approach is used in [21] to study a parabolic equation with a fractional Gaussian noise where the stochastic term is a nonlinear function of the solution. Strong solutions of bilinear evolution equations

* Received by the editors March 4, 2008; accepted for publication (in revised form) November 11, 2008; published electronically February 20, 2009. Research supported in part by NSF grants DMS 0204669, DMS 0505706, ANI 0124510, and GACR 201/04/0750.

http://www.siam.org/journals/sima/40-6/71764.html

$\dagger$ Department of Mathematics, University of Kansas, Lawrence, KS 66045 (duncan@math.ku.edu, bozenna@math.ku.edu).

${ }^{\ddagger}$ Institute of Mathematics, Czech Academy of Sciences, Prague, Czech Republic (maslow@math. cas.cz). 
with a fractional Brownian motion are considered in [11,12], and the same type of equation is studied in [33], where a fractional Feynman-Kac formula is obtained. A stochastic wave equation with a fractional Gaussian noise is considered in [2], and a stochastic heat equation with a multiparameter fractional Gaussian noise is studied in $[16,18]$.

One facet of the motivation for the study of weak solutions in an infinitedimensional space follows from some results $[7,27]$ for weak solutions in finitedimensional spaces that use an absolutely continuous transformation of measures which generalize the result of Girsanov [14] for Brownian motion.

In this paper, a similar analysis is made for infinite-dimensional state spaces. While the structure of the infinite-dimensional Girsanov theorem is analogous to the finite-dimensional case, significant distinct difficulties arise when the application of this theorem is used for stochastic equations in infinite-dimensional spaces. First the driving process is only cylindrical, so the Girsanov theorem can only be used to transform the semilinear equation to a linear equation that is a fractional OrnsteinUhlenbeck process. Since there is no classical strong solution to the linear equation, the mild solution must be used, making the analysis of the transformation of the measures by the Radon-Nikodým derivative more difficult because a suitable sample path regularity of the Ornstein-Uhlenbeck process must be verified. Unlike the finite-dimensional case, this regularity is not immediate and some assumptions on the coefficients in the linear equation must be made which are known for the case of Brownian motion. The sample regularity requirement increases as the Hurst parameter $H$ increases. Dually the operators that appear in the Radon-Nikodým derivative are less regular as $H$ increases. Thus the applicability of the Girsanov theorem is not immediate in this case and some conditions must be determined for the whole procedure to succeed. Furthermore, for $H>\frac{1}{2}$ in the finite-dimensional case it is assumed that the nonlinear term in the semilinear equation satisfies a global Hölder condition, but this assumption is not satisfied in many typical examples in infinite-dimensional spaces, such as reaction-diffusion equations. Thus this Hölder condition is relaxed here as well.

In section 2, some results from fractional calculus are given, and these results are used to describe a kernel function for an integral operator that provides an isometry of the second moment of Wiener-type stochastic integrals with respect to a fractional Brownian motion and the Lebesgue space of square integrable functions. Furthermore, some recent results for the solution of a linear stochastic equation in a Hilbert space [28] are described. In section 3, semilinear stochastic equations in a Hilbert space are studied. Initially, an absolute continuity of measures result for transforming the solution of a linear stochastic equation is verified that can be viewed as an analogue of the result of Girsanov [14] for a transformation of a finite-dimensional standard Brownian motion. For a semilinear stochastic equation where the nonlinear term satisfies a linear growth condition and some additional conditions are satisfied, it is shown that there is one and only one weak solution. The weak solution is obtained by verifying an absolute continuity of the measure of the solution with respect to the measure of the solution of the associated linear equation. The cases $H \in(0,1 / 2)$ and $H \in(1 / 2,1)$ are treated separately. Absolute continuity of the above measures is verified when the nonlinearity satisfies a power growth condition and some additional assumptions are made. In section 4, some examples of stochastic differential and partial differential equations are given that satisfy the assumptions of the theorems. 
2. Preliminaries. In this section, a cylindrical fractional Brownian motion in a separable Hilbert space is introduced, a Wiener-type stochastic integral with respect to this process is defined, and some basic properties of this integral are noted. Initially, some facts from the theory of fractional integration (cf. [31]) are described. Let $(V,\|\cdot\|,\langle\cdot, \cdot\rangle)$ be a separable Hilbert space. If $\varphi \in L^{1}([0, T], V)$, then for $\alpha>0$ the left-side and the right-side fractional (Riemann-Liouville) integrals of $\varphi$ are defined (for almost all $t \in[0, T]$ ) by

$$
\left(I_{0+}^{\alpha} \varphi\right)(t)=\frac{1}{\Gamma(\alpha)} \int_{0}^{t}(t-s)^{\alpha-1} \varphi(s) d s
$$

and

$$
\left(I_{T-}^{\alpha} \varphi\right)(t)=\frac{1}{\Gamma(\alpha)} \int_{t}^{T}(s-t)^{\alpha-1} \varphi(s) d s,
$$

respectively, where $\Gamma(\cdot)$ is the gamma function. For $\alpha \in(0,1)$ the inverse operators of these fractional integrals are called fractional derivatives and can be given by their respective Weyl representations

$$
\left(D_{0+}^{\alpha} \psi\right)(t)=\frac{1}{\Gamma(1-\alpha)}\left(\frac{\psi(t)}{t^{\alpha}}+\alpha \int_{0}^{t} \frac{\psi(t)-\psi(s)}{(t-s)^{\alpha+1}} d s\right)
$$

and

$$
\left(D_{T-}^{\alpha} \psi\right)(t)=\frac{1}{\Gamma(1-\alpha)}\left(\frac{\psi(t)}{(T-t)^{\alpha}}+\alpha \int_{t}^{T} \frac{\psi(s)-\psi(t)}{(s-t)^{\alpha+1}} d s\right),
$$

where $\psi \in I_{0+}^{\alpha}\left(L^{1}([0, T], V)\right)$ and $\psi \in I_{T-}^{\alpha}\left(L^{1}([0, T], V)\right)$, respectively.

Let $K_{H}(t, s)$ for $0 \leq s \leq t \leq T$ be the real-valued kernel function

$$
K_{H}(t, s)=\frac{\tilde{c}_{H}(t-s)^{H-\frac{1}{2}}}{\Gamma\left(H+\frac{1}{2}\right)}+\frac{\tilde{c}_{H}\left(\frac{1}{2}-H\right)}{\Gamma\left(H+\frac{1}{2}\right)} \int_{s}^{t}(u-s)^{H-\frac{3}{2}}\left(1-\left(\frac{s}{u}\right)^{\frac{1}{2}-H}\right) d u
$$

for $H \in(0,1 / 2)$. If $H \in(1 / 2,1)$, then $K_{H}$ has a simpler form as

$$
K_{H}(t, s)=\frac{\hat{c}_{H}}{\Gamma\left(H-\frac{1}{2}\right)} s^{\frac{1}{2}-H} \int_{s}^{t}(u-s)^{H-\frac{3}{2}} u^{H-\frac{1}{2}} d u .
$$

The terms $\tilde{c}_{H}$ and $\hat{c}_{H}$ are constants that depend only on $H$.

Define the integral operator $\mathbb{K}_{H}$ induced from the kernel $K_{H}$ by

$$
\mathbb{K}_{H} \varphi(t)=\int_{0}^{t} K_{H}(t, s) h(s) d s
$$

for $h \in L^{2}([0, T], V)$. It is well known [31] that

$$
\mathbb{K}_{H}: L^{2}([0, T], V) \rightarrow I_{0+}^{H+\frac{1}{2}}\left(L^{2}([0, T], V)\right)
$$

is a bijection and $\mathbb{K}_{H}$ can be described as

$$
\mathbb{K}_{H} h(s)=\bar{c}_{H} I_{0+}^{2 H}\left(u_{\frac{1}{2}-H} I_{0+}^{\frac{1}{2}-H}\left(u_{H-\frac{1}{2}} h\right)\right)(s)
$$

Copyright $@$ by SIAM. Unauthorized reproduction of this article is prohibited. 
for $H \in(0,1 / 2]$ and

$$
\mathbb{K}_{H} h(s)=c_{H} I_{0+}^{1}\left(u_{H-\frac{1}{2}} I_{0+}^{H-\frac{1}{2}}\left(u_{\frac{1}{2}-H} h\right)\right)(s)
$$

for $H \in[1 / 2,1)$, where

$$
\begin{gathered}
c_{H}=\left[\frac{2 H \Gamma\left(H+\frac{1}{2}\right) \Gamma\left(\frac{3}{2}-H\right)}{\Gamma(2-2 H)}\right]^{\frac{1}{2}}, \\
\bar{c}_{H}=c_{H} \Gamma(2 H),
\end{gathered}
$$

and

$$
u_{a}(s)=s^{a} I
$$

for $s \geq 0$ and $a \in \mathbb{R}$. The inverse operator

$$
\mathbb{K}_{H}^{-1}: I_{0+}^{H+\frac{1}{2}}\left(L^{2}([0, T], V)\right) \rightarrow L^{2}([0, T], V)
$$

is given by

$$
\mathbb{K}_{H}^{-1} \varphi(s)=\bar{c}_{H}^{-1} s^{\frac{1}{2}-H} D_{0+}^{\frac{1}{2}-H}\left(u_{H-\frac{1}{2}} D_{0+}^{2 H} \varphi\right)(s)
$$

for $H \in(0,1 / 2]$ and

$$
\mathbb{K}_{H}^{-1} \varphi(s)=c_{H}^{-1} s^{H-\frac{1}{2}} D_{0+}^{H-\frac{1}{2}}\left(u_{\frac{1}{2}-H} D \varphi\right)(s)
$$

for $H \in[1 / 2,1)$ and $\varphi \in I_{0+}^{H+\frac{1}{2}}\left(L^{2}([0, T], V)\right)$. Note that if $\varphi \in H^{1}([0, T], V)$, the Sobolev space, then

$$
\mathbb{K}_{H}^{-1} \varphi(s)=\bar{c}_{H}^{-1} s^{H-\frac{1}{2}} I_{0+}^{\frac{1}{2}-H}\left(u_{\frac{1}{2}-H} \varphi^{\prime}\right)(s)
$$

for $H \in(0,1 / 2]$.

Since the operator $\mathbb{K}_{H}^{-1}$ plays an important role in what follows, it is desirable to have some information about its domain $I_{0+}^{H+\frac{1}{2}}\left(L^{2}([0, T], V)\right)$. It is straightforward that $I_{0+}^{H+\frac{1}{2}}\left(L^{2}([0, T], V)\right) \subset C^{\beta}([0, T], V)$ for $\beta>\frac{1}{2}-H$ and $H \in(0,1 / 2)$. However, in section 3 , a more refined result is needed. If $H \in(1 / 2,1)$, then $I_{0+}^{H+\frac{1}{2}}\left(L^{2}([0, T], V)\right) \subset$ $L^{2}([0, T], V)$.

A definition of the stochastic integral of a deterministic $V$-valued function with respect to a scalar fractional Brownian motion $(\beta(t), t \geq 0)$ is given. This definition uses the methods in $[1,6,11,30]$. An alternative, equivalent method is given in [10].

A family of linear operators $\left(\mathcal{K}_{H}^{*}, H \in(0,1)\right)$ is defined which provides an isometry between Wiener-type integrals of a fractional Brownian motion and $L^{2}([0, T], V)$. It is written as an adjoint because the linear operator $\mathcal{K}_{H}$ occurs naturally in the factorization of the covariance for a fractional Brownian motion in $L^{2}$.

Let $\mathcal{K}_{H}^{*}: \mathcal{E} \rightarrow L^{2}([0, T], V)$ be the linear map given by

$$
\mathcal{K}_{H}^{*} \varphi(t)=\varphi(t) K_{H}(T, t)+\int_{t}^{T}(\varphi(s)-\varphi(t)) \frac{\partial K_{H}}{\partial s}(s, t) d s
$$

Copyright $@$ by SIAM. Unauthorized reproduction of this article is prohibited. 
for $\varphi \in \mathcal{E}$ and $K_{H}$ given by (2.1), where $\mathcal{E}$ is the linear space of $V$-valued step functions on $[0, T]$, that is, $\varphi \in \mathcal{E}$ if

$$
\varphi(t)=\sum_{i=1}^{n-1} x_{i} \mathbb{1}_{\left[t_{i}, t_{i+1}\right)}(t)
$$

where $x_{i} \in V$ for $i \in\{1, \ldots, n-1\}$ and $0=t_{1}<t_{2}<\cdots<t_{n}=T$.

Define the stochastic integral as

$$
\int_{0}^{T} \varphi d \beta:=\sum_{i=1}^{n} x_{i}\left(\beta\left(t_{i+1}\right)-\beta\left(t_{i}\right)\right) .
$$

It follows directly that

$$
\mathbb{E}\left\|\int_{0}^{T} \varphi d \beta\right\|^{2}=\left|\mathcal{K}_{H}^{*} \varphi\right|_{L^{2}([0, T], V)}^{2},
$$

where $|\cdot|_{L^{2}([0, T], V)}$ is the norm in $L^{2}([0, T], V)$ induced by the inner product. Let $\left(\mathcal{H},|\cdot|_{\mathcal{H}},\langle\cdot, \cdot\rangle_{\mathcal{H}}\right)$ be the Hilbert space obtained by the completion of the pre-Hilbert space $\mathcal{E}$ with the inner product

$$
\langle\varphi, \psi\rangle_{\mathcal{H}}:=\left\langle\mathcal{K}_{H}^{*} \varphi, \mathcal{K}_{H}^{*} \psi\right\rangle_{L^{2}([0, T], V)}
$$

for $\varphi, \psi \in \mathcal{E}$. The stochastic integral is extended to an arbitrary $\varphi \in \mathcal{H}$ by the isometry (2.11). Thus $\mathcal{H}$ is a linear space of integrable functions, and it is useful to obtain some more specific information about $\mathcal{H}$. If $H \in(1 / 2,1)$, then it is easily verified that $\mathcal{H} \supset \tilde{\mathcal{H}}$, where $\tilde{\mathcal{H}}$ is the Banach space of Borel measurable functions with the norm $|\cdot|_{\tilde{\mathcal{H}}}$ given by

$$
|\varphi|_{\tilde{\mathcal{H}}}^{2}:=\int_{0}^{T} \int_{0}^{T}\|\varphi(u)\|\|\varphi(v)\| \phi_{H}(u-v) d u d v
$$

where $\phi_{H}(u)=H(2 H-1)|u|^{2 H-2}$, and it can be verified that $\tilde{\mathcal{H}} \supset L^{\frac{1}{H}}([0, T], V)$ and, in particular, $\tilde{\mathcal{H}} \supset L^{2}([0, T], V)$ (cf. [12]). If $\varphi \in \tilde{\mathcal{H}}$ and $H>1 / 2$, then

$$
\mathbb{E}\left\|\int_{0}^{T} \varphi d \beta\right\|^{2}=\int_{0}^{T} \int_{0}^{T}\langle\varphi(u), \varphi(v)\rangle \phi_{H}(u-v) d u d v .
$$

If $H \in(0,1 / 2)$, then the space of integrable functions is smaller than for $H \in$ $(1 / 2,1)$. For $H \in(0,1 / 2)$ it is known that $\mathcal{H} \supset H^{1}([0, T], V)$ (cf. [17, Theorem 5.20]) and $\mathcal{H} \supset C^{\beta}([0, T], V)$ for each $\beta>1 / 2-H$ (a more specific result is given in the next section). If $H \in(0,1 / 2)$, then the linear operator $\mathcal{K}_{H}^{*}$ can be described by a fractional derivative

$$
\mathcal{K}_{H}^{*} \varphi(t)=c_{H} t^{\frac{1}{2}-H} D_{T-}^{\frac{1}{2}-H}\left(u_{H-\frac{1}{2}} \varphi\right)(t),
$$

where its domain is $\mathcal{H}=I_{T-}^{\frac{1}{2}-H}\left(L^{2}([0, T], V)\right)$ (cf. [1, Proposition 6]). If $H \in(1 / 2,1)$, then

$$
\mathcal{K}_{H}^{*} \varphi(t)=c_{H} t^{\frac{1}{2}-H} I_{T-}^{H-\frac{1}{2}}\left(u_{H-\frac{1}{2}} \varphi\right)(t)
$$

Copyright (C) by SIAM. Unauthorized reproduction of this article is prohibited. 
A standard cylindrical fractional Brownian motion is defined now.

Definition 2.1. Let $(\Omega, \mathcal{F}, \mathbb{P})$ be a complete probability space. A cylindrical process $\langle B, \cdot\rangle: \Omega \times \mathbb{R}_{+} \times V \rightarrow \mathbb{R}$ on $(\Omega, \mathcal{F}, \mathbb{P})$ is called a standard cylindrical fractional Brownian motion with the Hurst parameter $H \in(0,1)$ if

(1) for each $x \in V \backslash\{0\}, \frac{1}{\|x\|}\langle B(\cdot), x\rangle$ is a standard scalar fractional Brownian motion with the Hurst parameter $H$;

(2) for $\alpha, \beta \in \mathbb{R}$ and $x, y \in V$

$$
\langle B(t), \alpha x+\beta y\rangle=\alpha\langle B(t), x\rangle+\beta\langle B(t), y\rangle \quad \text { a.s. } \mathbb{P} .
$$

Note that $\langle B(t), x\rangle$ has the interpretation of the evaluation of the functional $B(t)$ at $x$ though the process $B(\cdot)$ does not take values in $V$.

For $H=1 / 2$, this definition is the usual one for a standard cylindrical Wiener process in $V$. For a complete orthonormal basis $\left(e_{n}, n \in \mathbb{N}\right)$ of $V$, letting $\beta_{n}(t)=$ $\left\langle B(t), e_{n}\right\rangle$ for $n \in \mathbb{N}$, the sequence of scalar processes $\left(\beta_{n}, n \in \mathbb{N}\right)$ is independent and $B$ can be represented by the formal series

$$
B(t)=\sum_{n=1}^{\infty} \beta_{n}(t) e_{n}
$$

that does not converge a.s. in $V$.

Naturally associated with a standard cylindrical fractional Brownian motion is a standard cylindrical Wiener process $(W(t), t \geq 0)$ in $V$ such that, formally, $\dot{B}(t)=$ $\mathcal{K}_{H} \dot{W}(t)$. For $x \in V \backslash\{0\}$, let $\beta_{x}(t)=\langle B(t), x\rangle$. It is elementary to verify from (2.1) that there is a scalar Wiener process $\left(w_{x}(t), t \geq 0\right)$ such that

$$
\beta_{x}(t)=\int_{0}^{t} K_{H}(t, s) d w_{x}(s)
$$

for $t \in \mathbb{R}_{+}$. Dually, $w_{x}(t)=\beta_{x}\left(\left(\mathcal{K}_{H}^{*}\right)^{-1} \mathbb{1}_{[0, t)}\right)$, where $\mathcal{K}_{H}^{*}$ is given by $(2.15)$ or $(2.16)$ and $V=\mathbb{R}$. Thus there is a formal expansion of $W$,

$$
W(t)=\sum_{n=1}^{\infty} w_{n}(t) e_{n}
$$

where $\left(e_{n}, n \in \mathbb{N}\right)$ is a complete orthonormal basis for $V$ and $w_{n}=\left\langle W, e_{n}\right\rangle$ for $n \in \mathbb{N}$.

Now, the stochastic integral $\int_{0}^{T} G d B$ is defined for a suitable operator-valued function $G:[0, T] \rightarrow \mathcal{L}(V)$ so that the integral is a $V$-valued random variable.

Definition 2.2. Let $G:[0, T] \rightarrow \mathcal{L}(V)$ be Borel measurable, let $\left(e_{n}, n \in \mathbb{N}\right)$ be a complete orthonormal basis in $V$, let $G(\cdot) e_{n} \in \mathcal{H}$ for each $n \in \mathbb{N}$, and let $B$ be a standard cylindrical fractional Brownian motion for some fixed $H \in(0,1)$. The stochastic integral $\int_{0}^{T} G d B$ is defined as

$$
\int_{0}^{T} G d B:=\sum_{n=1}^{\infty} \int_{0}^{T} G e_{n} d \beta_{n}
$$

provided the infinite series converges in $L^{2}(\Omega, V)$.

It is elementary to verify that this definition does not depend on the complete orthonormal basis that is used.

Copyright $\odot$ by SIAM. Unauthorized reproduction of this article is prohibited. 
The following proposition describes some $\mathcal{L}(V)$-valued functions $G$ that can be used as integrands in Definition 2.2.

Proposition 2.3. Let $G:[0, T] \rightarrow \mathcal{L}(V)$ be Borel measurable and let $G(\cdot) x \in \mathcal{H}$ for each $x \in V$. Let $\Gamma_{T}: V \rightarrow L^{2}([0, T], V)$ be given as

$$
\left(\Gamma_{T} x\right)(t)=\left(\mathcal{K}_{H}^{*} G x\right)(t)
$$

for $t \in[0, T]$ and $x \in V$. If $\Gamma_{T} \in \mathcal{L}_{2}\left(V, L^{2}([0, T], V)\right)$, the linear space of HilbertSchmidt operators, then the stochastic integral (2.20) is a centered Gaussian V-valued random variable with the covariance operator $\tilde{Q}_{T}$ given by

$$
\tilde{Q}_{T} x=\int_{0}^{T} \sum_{n=1}^{\infty}\left\langle\left(\Gamma_{T} e_{n}\right)(s), x\right\rangle\left(\Gamma_{T} e_{n}\right)(s) d s .
$$

This integral does not depend on the choice of the complete orthonormal basis $\left(e_{n}, n \in\right.$ $\mathbb{N})$ in $V$.

Proof. Substituting $G$ in the definition of the stochastic integral (2.20), it is clear that the terms of the summation on the right-hand side are $V$-valued Gaussian random variables by the construction of the integral for a scalar fractional Brownian motion, and the sequence of random variables $\left(\int_{0}^{T} G e_{n} d \beta_{n}, n \in \mathbb{N}\right)$ is independent. Computing the second moment of the tail of the series in (2.20) yields

$$
\begin{aligned}
\mathbb{E}\left\|\sum_{k=m}^{\infty} G e_{k} d \beta_{k}\right\|^{2}=\sum_{k=m}^{\infty} \mathbb{E}\left\|\int_{0}^{T} G e_{k} d \beta_{k}\right\|^{2}=\sum_{k=m}^{\infty} \int_{0}^{T}\left\|\left(\mathcal{K}_{H}^{*} G e_{k}\right)(s)\right\|^{2} d s \\
=\sum_{k=m}^{\infty} \int_{0}^{T}\left\|\left(\Gamma_{T} e_{k}\right)(s)\right\|^{2} d s=\sum_{k=m}^{\infty}\left|\Gamma_{T} e_{k}\right|_{L^{2}([0, T], V)}^{2} .
\end{aligned}
$$

It is clear that this final series tends to zero as $m$ tends to infinity. Thus there is convergence in $L^{2}(\Omega, V)$ of the partial sums of the infinite series in (2.20).

To verify that (2.20) is a Gaussian random variable and the form of the covariance $\tilde{Q}_{T}$, initially note that for any $\varphi \in \mathcal{H}$ and $x \in V$, there is the equality

$$
\int_{0}^{T} \varphi d \beta_{x}=\int_{0}^{T} \mathcal{K}_{H}^{*} \varphi d w_{x}
$$

where $w_{x}$ is the Wiener process given by (2.18). The terms in the infinite series on the right-hand side of (2.20) are $V$-valued, independent centered Gaussian random variables with the sequence of covariance operators $\left(\tilde{Q}_{T}^{(n)}, n \in \mathbb{N}\right)$

$$
\tilde{Q}_{T}^{(n)} x=\int_{0}^{T}\left\langle\left(\mathcal{K}_{H}^{*} G e_{n}\right)(s), x\right\rangle\left(\mathcal{K}_{H}^{*} G e_{n}\right)(s) d s
$$

for each $n \in \mathbb{N}$ and $x \in V$. Thus

$$
\begin{aligned}
\tilde{Q}_{T} x=\sum_{n=1}^{\infty} \int_{0}^{T}\left\langle\left(\mathcal{K}_{H}^{*} G e_{n}\right)(s), x\right\rangle\left(\mathcal{K}_{H}^{*} G e_{n}\right)(s) d s \\
\quad=\int_{0}^{T} \sum_{n=1}^{\infty}\left\langle\left(\Gamma_{T} e_{n}\right)(s), x\right\rangle\left(\Gamma_{T} e_{n}\right)(s) d s .
\end{aligned}
$$

Copyright $\odot$ by SIAM. Unauthorized reproduction of this article is prohibited. 
The summability of the infinite series on the right-hand side follows from the HilbertSchmidt property of $\Gamma_{T}$. The independence of the stochastic integral from the choice of the complete orthonormal basis follows from (2.23) and the analogous property for stochastic integrals with respect to a standard cylindrical Wiener process.

Since for almost all $t \in[0, T]$ the linear operator $\Gamma_{T}(\cdot)(t): V \rightarrow V$ is HilbertSchmidt, so we denote for almost all $t \in[0, T]$ the adjoint of $\Gamma_{T}(\cdot)(t)$ as $\Gamma_{T}^{*}(\cdot)(t)$ : $V \rightarrow V$. It follows by (2.25) that for $x, y \in V$,

$$
\begin{aligned}
\left\langle\tilde{Q}_{T} x, y\right\rangle & =\int_{0}^{T} \sum_{n=1}^{\infty}\left\langle\left(\Gamma_{T} e_{n}\right)(s), x\right\rangle\left\langle\left(\Gamma_{T} e_{n}\right)(s), y\right\rangle d s \\
& =\int_{0}^{T} \sum_{n=1}^{\infty}\left\langle e_{n},\left(\Gamma_{T}^{*} x\right)(s)\right\rangle\left\langle e_{n},\left(\Gamma_{T}^{*} y\right)(s)\right\rangle d s \\
& =\int_{0}^{T}\left\langle\left(\Gamma_{T}^{*} x\right)(s),\left(\Gamma_{T}^{*} y\right)(s)\right\rangle d s \\
& =\int_{0}^{T}\left\langle\Gamma_{T} \Gamma_{T}^{*} x(s), y\right\rangle d s .
\end{aligned}
$$

If $H \in(1 / 2,1)$, then $\tilde{Q}_{T}$ satisfies

$$
\tilde{Q}_{T}=\int_{0}^{T} \int_{0}^{T} G(u) G^{*}(v) \phi_{H}(u-v) d u d v
$$

where $\phi_{H}(u)=H(2 H-1)|u|^{2 H-2}$ and $G$ is assumed to satisfy

$$
\int_{0}^{T} \int_{0}^{T}|G(u)|_{\mathcal{L}_{2}(V)}|G(v)|_{\mathcal{L}_{2}(V)} \phi_{H}(u-v) d u d v<\infty
$$

(cf. [13, Proposition 2.2]).

The next proposition shows that some densely defined linear operators commute with the stochastic integration.

Proposition 2.4. If $\tilde{A}: \operatorname{Dom}(\tilde{A}) \rightarrow V$ is a closed linear operator, $\operatorname{Dom}(\tilde{A}) \subset V$, and $G:[0, T] \rightarrow \mathcal{L}(V)$ is Borel measurable such that $G([0, T]) \subset \operatorname{Dom}(\tilde{A})$ and both $G$ and $\tilde{A} G$ satisfy the conditions for $G$ in Proposition 2.3, then

$$
\int_{0}^{T} G d B \subset \operatorname{Dom}(\tilde{A}) \quad \text { a.s. } \mathbb{P}
$$

and

$$
\tilde{A} \int_{0}^{T} G d B=\int_{0}^{T} \tilde{A} G d B \quad \text { a.s. } \mathbb{P} .
$$

Proof. By the assumptions on $G$ and $\tilde{A} G$, it follows that $G e_{n} \in \mathcal{H}$ and $\tilde{A} G e_{n} \in \mathcal{H}$ for $n \in \mathbb{N}$, so by a standard argument using a sequence of step function integrands, the following equality is satisfied:

$$
\tilde{A} \int_{0}^{T} G e_{n} d \beta_{n}=\int_{0}^{T} \tilde{A} G e_{n} d \beta_{n} .
$$

Copyright (c) by SIAM. Unauthorized reproduction of this article is prohibited. 
Since the sequence of integrals that are obtained from a complete orthonormal basis $\left(e_{n}, n \in \mathbb{N}\right)$ are Gaussian random variables it follows that

$$
\lim _{m \rightarrow \infty} \sum_{n=1}^{m} \int_{0}^{T} G e_{n} d \beta_{n}=\int_{0}^{T} G d B \quad \text { a.s. } \mathbb{P}
$$

and

$$
\lim _{m \rightarrow \infty} \tilde{A}\left(\sum_{n=1}^{m} \int_{0}^{T} G e_{n} d \beta_{n}\right)=\lim _{m \rightarrow \infty} \sum_{n=1}^{m} \int_{0}^{T} \tilde{A} G e_{n} d \beta_{n}=\int_{0}^{T} \tilde{A} G d B \quad \text { a.s. } \mathbb{P} .
$$

Since $\tilde{A}$ is a closed linear operator it follows that $\int_{0}^{T} G d B \in \operatorname{Dom}(\tilde{A})$ a.s. $\mathbb{P}$ and equality (2.26) is satisfied.

Some results are reviewed for a linear stochastic differential equation with a cylindrical fractional Brownian motion whose solution is often called a fractional OrnsteinUhlenbeck process. This process is a mild solution of the linear stochastic equation

$$
\begin{aligned}
d Z(t) & =A Z(t) d t+\Phi d B(t), \\
Z(0) & =x,
\end{aligned}
$$

where $Z(t), x \in V,(B(t), t \geq 0)$ is a standard cylindrical fractional Brownian with $H \in(0,1), \Phi \in \mathcal{L}(V), A: \operatorname{Dom}(A) \rightarrow V$, $\operatorname{Dom}(A) \subset V$, and $A$ is the infinitesimal generator of a strongly continuous semigroup $(S(t), t \geq 0)$ on $V$. A mild solution of $(2.28)$ is

$$
\begin{aligned}
Z(t) & =S(t) x+\int_{0}^{t} S(t-r) \Phi d B(r) \\
& =S(t) x+\hat{Z}(t),
\end{aligned}
$$

where the stochastic integral in (2.29) is given by Definition 2.2 .

Typically it is assumed that $(S(t), t \geq 0)$ is an analytic semigroup. In this case, there is a $\hat{\beta} \in \mathbb{R}$ such that the operator $\hat{\beta} I-A$ is uniformly positive on $V$; that is, the resolvent set contains $\{\lambda \in \mathbb{C} ;|\arg \lambda|<\pi / 2+\delta\} \cup U$, where $\delta>0$ and $U$ is a neighborhood of zero.

For each $\delta \geq 0,\left(V_{\delta},\|\cdot\|_{\delta}\right)$ is a Hilbert space where $V_{\delta}=\operatorname{Dom}\left((\hat{\beta} I-A)^{\delta}\right)$ with the graph norm topology so that

$$
\|x\|_{\delta}=\left\|(\hat{\beta} I-A)^{\delta} x\right\| .
$$

For the mild solution of $(2.28)$, the cases $H \in(0,1 / 2)$ and $H \in(1 / 2,1)$ have been treated separately $[13,28]$ because the conditions for similar results are somewhat different. The case $H=1 / 2$ (Brownian motion) has been studied extensively (cf. [4]). in [13].

For $H \in(1 / 2,1)$, the following sample path property of the solution is described

Proposition 2.5. If $H \in(1 / 2,1), S(t) \Phi \in \mathcal{L}_{2}(V)$ for each $t>0$ and

$$
\int_{0}^{T_{0}} \int_{0}^{T_{0}} u^{-\alpha} v^{-\alpha}|S(u) \Phi|_{\mathcal{L}_{2}(V)}|S(v) \Phi|_{\mathcal{L}_{2}(V)} \phi_{H}(u-v) d u d v<\infty
$$

Copyright (C) by SIAM. Unauthorized reproduction of this article is prohibited. 
for some $T_{0}>0$ and $\alpha>0$, then there is a Hölder continuous $V$-valued version of the process $(\hat{Z}(t), t \geq 0)$ with Hölder exponent $\beta<\alpha$, where $\hat{Z}$ is the stochastic convolution in (2.29) and $\phi_{H}$ is given in (2.13). If $(S(t), t \geq 0)$ is an analytic semigroup, then there is a version of the process $(\hat{Z}(t), t \in[0, T])$ with $C^{\beta}\left([0, T], V_{\delta}\right)$ sample paths for each $T>0$ and $\beta+\delta<\alpha$.

For each $H \in(0,1)$, there are the following results for the sample path behavior of the mild solution [28].

Proposition 2.6. Let $(S(t), t \geq 0)$ be an analytic semigroup, let $H \in(0,1)$, and let

$$
|S(t) \Phi|_{\mathcal{L}_{2}(V)} \leq c t^{-\gamma}
$$

for $t \in[0, T]$, some $c>0$, and $\gamma \in[0, H)$. Let $\alpha \geq 0$ and $\delta \geq 0$ satisfy

$$
\alpha+\delta+\gamma<H
$$

Then there is a version of the process $(\hat{Z}(t), t \in[0, T])$ with $C^{\alpha}\left([0, T], V_{\delta}\right)$ sample paths. If it is assumed instead of (2.31) and (2.32) that $\Phi \in \mathcal{L}_{2}(V)$ and $\alpha+\delta<H$, then the process $(\hat{Z}(t), t \in[0, T])$ has a $C^{\alpha}\left([0, T], V_{\delta}\right)$ version. In particular, there is a $C^{\alpha}([0, T], V)$ version for $0<\alpha<H$.

3. Semilinear stochastic equations. In this section, both weak and mild solutions are obtained for various semilinear stochastic equations with a fractional Brownian motion. The cases $H \in(0,1 / 2)$ and $H \in(1 / 2,1)$ are treated separately as in the case of the linear stochastic equations (Propositions 2.5 and 2.6). The weak solution of a semilinear equation is obtained by an absolutely continuous transformation of the measure for the solution of the associated linear equation. The absolute continuity methods given here are an analogue of the results for the measure of a finitedimensional fractional Brownian motion $[7,9,25,26]$ and the results for Wiener measure [3,14]. For a fixed $H \in(0,1)$ and $T>0$, let $\left(\mathcal{F}_{t}, t \in[0, T]\right)$ be the filtration for the standard cylindrical fractional Brownian motion $(B(t), t \in[0, T])$ with the Hurst parameter $H$. The sub- $\sigma$-algebra $\mathcal{F}_{t} \subset \mathcal{F}$ can be generated by $\sigma\left(\beta_{n}(s), s \in[0, t], n \in \mathbb{N}\right)$, where $\left(\beta_{n}, n \in \mathbb{N}\right)$ is a sequence of independent scalar fractional Brownian motions with the Hurst parameter $H$ that is given in the definition of a standard cylindrical fractional Brownian motion (Definition 2.1).

The following result describes an absolute continuity for a transformation of a standard cylindrical fractional Brownian motion.

TheOREM 3.1. Let $H \in(0,1)$ and $T>0$ be fixed and let $(u(t), t \in[0, T])$ be a $V$-valued, $\left(\mathcal{F}_{t}\right)$-adapted process such that

1.

$$
\int_{0}^{T}\|u(t)\| d t<\infty \quad \text { a.s. } \mathbb{P}
$$

and

2 .

$$
U(\cdot):=\int_{0}^{\cdot} u(s) d s \in I_{0+}^{H+\frac{1}{2}}\left(L^{2}([0, T], V)\right) \quad \text { a.s. } \mathbb{P} .
$$

Furthermore, it is assumed that

$$
\mathbb{E} \xi(T)=1,
$$

Copyright (c) by SIAM. Unauthorized reproduction of this article is prohibited. 
where

$$
\xi(T)=\exp \left[\int_{0}^{T}\left\langle\mathbb{K}_{H}^{-1}(U)(t), d W(t)\right\rangle-\frac{1}{2} \int_{0}^{T}\left\|\mathbb{K}_{H}^{-1}(U)(t)\right\|^{2} d t\right],
$$

where $(W(t), t \in[0, T])$ is a standard cylindrical Wiener process in $V$ given by $(2.19)$ and $\mathbb{K}_{H}^{-1}$ is the inverse of the integral operator $\mathbb{K}_{H}$ in (2.3). Then the process $(\tilde{B}(t), t \in$ $[0, T])$ given by

$$
\tilde{B}(t):=B(t)-U(t)
$$

is a standard cylindrical fractional Brownian motion in $V$ with the Hurst parameter $H$ on the probability space $(\Omega, \mathcal{F}, \tilde{\mathbb{P}})$, where

$$
\frac{d \tilde{\mathbb{P}}}{d \mathbb{P}}=\xi(T) \quad \text { a.s. }
$$

Proof. Initially, it is noted that for an $\left(\mathcal{F}_{t}\right)$-adapted process, $(\eta(t), t \in[0, T])$ with $\eta \in L^{2}([0, T], V)$ a.s. $\mathbb{P}, \int_{0}^{T}\langle\eta, d W\rangle$ is defined by

$$
\int_{0}^{T}\langle\eta, d W\rangle=\sum_{n=1}^{\infty} \int_{0}^{T}\left\langle\eta, e_{n}\right\rangle d w_{n},
$$

where the sequences $\left(\beta_{n}, n \in \mathbb{N}\right)$ and $\left(w_{n}, n \in \mathbb{N}\right)$ are related by (2.18). It is shown that $\mathbb{K}_{H}^{-1} U$ satisfies the conditions of $\eta$ so that the stochastic integral in (2.20) is well-defined. Recall that the linear operator $\mathbb{K}_{H}$ given in (2.3) is a bijection

$$
\mathbb{K}_{H}: L^{2}([0, T], V) \rightarrow I_{0+}^{H+\frac{1}{2}}\left(L^{2}([0, T], V)\right),
$$

so by assumption 1 in Theorem $3.1, \mathbb{K}_{H}^{-1}(U) \in L^{2}([0, T], V)$ a.s. $\mathbb{P}$. From the definition of $\mathbb{K}_{H}$, it follows that $\left(\mathbb{K}_{H}^{-1}(U)(t), t \in[0, T]\right)$ is an $\left(\mathcal{F}_{t}\right)$-adapted process because $U$ is $\left(\mathcal{F}_{t}\right)$-adapted. By the construction of the standard cylindrical Wiener process $W$, it is a Wiener process with respect to $\left(\mathcal{F}_{t}\right)$ so $\xi_{T}$ is a well-defined random variable. By a Girsanov theorem for Wiener processes in infinite dimensions (cf. [4, 24]), equality (3.2) defines a probability $\tilde{\mathbb{P}}$ on $(\Omega, \mathcal{F})$ such that

$$
\tilde{W}(t):=W(t)-\int_{0}^{t} \mathbb{K}_{H}^{-1}(U)(s) d s
$$

is a standard cylindrical Wiener process in $V$. Let

$$
\tilde{\beta}_{n}(t):=\left\langle B(t), e_{n}\right\rangle-\left\langle U(t), e_{n}\right\rangle
$$

and

$$
\tilde{w}_{n}(t)=\left\langle W(t), e_{n}\right\rangle-\left\langle\int_{0}^{t} \mathbb{K}_{H}^{-1}(U)(s) d s, e_{n}\right\rangle
$$

It follows that

$$
\begin{aligned}
\int_{0}^{t} K_{H}(t, s) d \tilde{w}_{n}(s) & =\int_{0}^{t} K_{H}(t, s) d w_{n}(s)-\int_{0}^{t} K_{H}(t, s)\left\langle\mathbb{K}_{H}^{-1}(U)(s), e_{n}\right\rangle d s \\
& =\beta_{n}(t)-\left\langle\int_{0}^{t} K_{H}(t, s)\left(\mathbb{K}_{H}^{-1}(U)(s)\right) d s, e_{n}\right\rangle \\
& =\beta_{n}(t)-\left\langle\mathbb{K}_{H} \mathbb{K}_{H}^{-1}(U)(t), e_{n}\right\rangle \\
& =\beta_{n}(t)-\left\langle U(t), e_{n}\right\rangle=\tilde{\beta}_{n}(t) .
\end{aligned}
$$

Copyright (c) by SIAM. Unauthorized reproduction of this article is prohibited. 
Thus $(\tilde{B}(t), t \in[0, T])$ is a standard cylindrical fractional Brownian motion in $V$ with the Hurst parameter $H$ on $(\Omega, \mathcal{F}, \tilde{\mathbb{P}})$.

In this section, the following semilinear stochastic equation is considered:

$$
d X(t)=(A X(t)+F(X(t))) d t+\Phi d B(t),
$$

where $t \in \mathbb{R}_{+}, X(t), X_{0} \in V$ is nonrandom, $(B(t), t \geq 0)$ is a standard cylindrical fractional Brownian motion with the Hurst parameter $H \in(0,1), \Phi \in \mathcal{L}(V)$, $A: \operatorname{Dom}(A) \rightarrow V, \operatorname{Dom}(A) \subset V$, and $A$ is the infinitesimal generator of a strongly continuous semigroup $(S(t), t \geq 0)$ on $V$. The function $F: V \rightarrow V$ is nonlinear, and for the applications to stochastic partial differential equations it is more useful to assume that $F$ is defined only on a (dense) subspace of $V$. So, let $\left(E,\|\cdot\|_{E}\right)$ be a separable Banach space that is continuously embedded in $V$ and $F: E \rightarrow E$ with $X_{0} \in E$. Subsequently, it is assumed that $F: E \rightarrow E$ is Borel measurable, $\operatorname{Im}(F) \subset \operatorname{Im}(\Phi)$, for $G:=\Phi^{-1} F, G: E \rightarrow V$, and

$$
\|G(x)\| \leq \hat{k}\left(1+\|x\|_{E}^{\rho}\right)
$$

and

$$
\|F(x)\|_{E} \leq \hat{k}\left(1+\|x\|_{E}^{\rho}\right)
$$

for each $x \in E$ and some $\rho \geq 1$. Furthermore, it is assumed that there is a constant $\bar{K}$ such that for each pair $(x, y)$ in $\operatorname{Dom}(A)$, there is a $z^{*} \in \partial\|z\|_{E}$ such that

$$
\left\langle A x-A y+F(x)-F(y), z^{*}\right\rangle_{E, E^{*}} \leq \bar{K}\|x-y\|_{E},
$$

where $\partial\|z\|_{E}$ is the subdifferential of the norm $\|z\|_{E}$ at the point $z=x-y$ and $\langle\cdot, \cdot,\rangle_{E, E^{*}}$ is the pairing between $E$ and $E^{*}$. The basic results on subdifferentials can be found in [32]. Inequality (3.7) is a one-sided growth condition that ensures the absence of explosions of solutions of (3.4) in a finite time. Some subsequent examples should clarify its interpretation.

The notions of a weak and a mild solution of (3.4) are given now.

Definition 3.2. A weak solution of $(3.4)$ is a triple $\left(X(t), B(t),\left(\tilde{\Omega}, \tilde{\mathcal{F}},\left(\tilde{\mathcal{F}}_{t}\right), \tilde{\mathbb{P}}\right)\right.$, $t \geq 0)$, where $(B(t), t \geq 0)$ is a standard cylindrical fractional Brownian motion in $V$ that is defined on the probability space $(\tilde{\Omega}, \tilde{\mathcal{F}}, \tilde{\mathbb{P}}),(B(t), t \geq 0)$ and $(X(t), t \geq 0)$ are adapted to the filtration $\left(\tilde{\mathcal{F}}_{t}\right)$, and $(X(t), t \geq 0)$ is an E-valued process satisfying

$$
X(t)=S(t) X_{0}+\int_{0}^{t} S(t-r) F(X(r)) d r+\int_{0}^{t} S(t-r) \Phi d B(r) .
$$

A mild solution, $(X(t), t \geq 0)$ of (3.4), is an E-valued process on a fixed probability space $\left(\Omega, \mathcal{F},\left(\mathcal{F}_{t}\right), \mathbb{P}\right)$ with a given standard cylindrical fractional Brownian motion that is the fractional Brownian motion in $(3.8),(B(t), t \geq 0)$ and $(X(t), t \geq 0)$ are adapted to the filtration $\left(\mathcal{F}_{t}\right)$, and the process $(X(t), t \geq 0)$ satisfies $(3.8)$.

Equation (3.8) has a unique weak solution if, for any two weak solutions $(X(t)$, $\left.B(t),\left(\Omega, \mathcal{F},\left(\mathcal{F}_{t}\right), \mathbb{P}\right), t \geq 0\right)$ and $\left(\tilde{X}(t), \tilde{B}(t),\left(\tilde{\Omega}, \tilde{\mathcal{F}},\left(\tilde{\mathcal{F}}_{t}\right), \tilde{\mathbb{P}}\right), t \geq 0\right)$, the processes $(X(t)$, $t \geq 0)$ and $(\tilde{X}(t), t \geq 0)$ have the same probability law.

The equation has a unique mild solution if, for any two processes $\left(X_{1}(t), t \geq 0\right)$ and $\left(X_{2}(t), t \geq 0\right)$ that satisfy $(3.8)$ on the same probability space $\left(\Omega, \mathcal{F},\left(\mathcal{F}_{t}\right), \mathbb{P}\right)$ with the same standard cylindrical fractional Brownian motion, $\mathbb{P}\left(X_{1}(t)=X_{2}(t), t \geq 0\right)=1$.

Copyright (c) by SIAM. Unauthorized reproduction of this article is prohibited. 
A primary goal in this section is to verify weak existence and weak uniqueness of a solution of (3.4). Note that (H1) alone is not sufficient to ensure that the stochastic convolution has values in $E$. While the assumption (H2) is given in a rather general form, it is verified for particular examples in section 4 . Since the cases $H \in(0,1 / 2)$ and $H \in(1 / 2,1)$ require different methods, they are treated separately.

The following three assumptions are made to construct a solution of (3.4):

(H1) The semigroup $(S(t), t \geq 0)$ generated by $A$ is analytic on $V$ and for each $t \geq$ $0,\left.S(t)\right|_{E} \in \mathcal{L}(E)$ and $\left\|\left.S(t)\right|_{E}\right\|_{\mathcal{L}(E)}$ is bounded on compact time intervals.

(H2) $\Phi \in \mathcal{L}(V)$ is injective and for $T>0$ the stochastic convolution process

$$
\left(\int_{0}^{t} S(t-r) \Phi d B(r), t \in[0, T]\right)
$$

has a version with $C([0, T], E)$ sample paths.

(H3) The function $F: E \rightarrow V$ in (3.4) is Borel measurable, $\operatorname{Im}(F) \subset \operatorname{Im}(\Phi)$, and the function $G=\Phi^{-1} F: E \rightarrow V$ satisfies

$$
\|G(x)\| \leq k\left(1+\|x\|_{E}\right)
$$

for some $k>0$ and all $x \in E$.

The following result verifies a weak solution for $H \in(0,1 / 2)$.

THeOREM 3.3. If $H \in(0,1 / 2)$ and the conditions (H1)-(H3) are satisfied, then the semilinear equation (3.4) has a weak solution. If additionally $F: E \rightarrow E$ and

$$
\|F(x)\|_{E} \leq k_{1}\left(1+\|x\|_{E}\right)
$$

for some $k_{1}>0$ and all $x \in E$, then the weak solution is unique.

Proof. Initially, existence of a weak solution is verified. By a standard method that has been used for equations of the form (3.4) with a standard cylindrical Brownian motion (cf. $[4,24])$, it suffices to verify that the cylindrical process

$$
\tilde{B}(t)=B(t)-\int_{0}^{t} G(Z(s)) d s
$$

is a standard cylindrical fractional Brownian motion in a suitable probability space where

$$
Z(t)=S(t) X_{0}+\tilde{Z}(t)
$$

satisfies the associated linear equation. To use Theorem 3.1 it is necessary to verify that $G=\Phi^{-1} F$ satisfies the conditions of $U$ in this theorem, that is,

$$
\int_{0}^{\cdot} G(Z(s)) d s \in I_{0+}^{H+\frac{1}{2}}\left(L^{2}([0, T], V)\right)
$$

and

$$
\mathbb{E} \exp [\rho(Z)]=1,
$$

where

$$
\left.\rho(Z)=\int_{0}^{T}\left\langle\mathbb{K}_{H}^{-1}\left(\int_{0}^{.} G(Z)\right)\right)(t), d W(t)\right\rangle-\frac{1}{2} \int_{0}^{T}\left\|\mathbb{K}_{H}^{-1}\left(\int_{0} G(Z)\right)(t)\right\|^{2} d t,
$$


$\mathbb{K}_{H}^{-1}$ is the inverse of $\mathbb{K}_{H}$ in $(2.3)$, and $(W(t), t \geq 0)$ is a standard cylindrical Wiener process in $V$ by (2.19).

From (2.9), it follows that

$$
\begin{aligned}
\mid \mathbb{K}_{H}^{-1} & \left.\left(\int_{0} G(Z)\right)\right|_{L^{2}([0, T], V)} ^{2} \\
= & \bar{c}_{H}^{-2}\left|u_{H-\frac{1}{2}} I_{0+}^{\frac{1}{2}-H}\left(u_{\frac{1}{2}-H} G(Z)\right)\right|_{L^{2}([0, T], V)}^{2} \\
= & \hat{c}_{H} \int_{0}^{T}\left(s^{H-\frac{1}{2}}\left\|\int_{0}^{s} r^{\frac{1}{2}-H}(s-r)^{-\frac{1}{2}-H} G(Z(r)) d r\right\|\right)^{2} d s \\
\leq & \hat{c}_{H} k^{2}\left(1+|\tilde{Z}|_{C([0, T], E)}+\sup _{t \in[0, T]}\left\|S(t) X_{0}\right\|_{E}\right)^{2} \int_{0}^{T} s^{2 H-1} \\
& \cdot\left(\int_{0}^{s} r^{\frac{1}{2}-H}(s-r)^{-\frac{1}{2}-H} d r\right)^{2} d s \\
\leq & c_{T}\left(1+|\tilde{Z}|_{C([0, T], E)}^{2}\right)
\end{aligned}
$$

for some $c_{T}>0$ that depends only on $T$. This inequality verifies (3.11). By (3.14) it follows directly that

$$
\mathbb{E} \exp \left[\hat{k} \int_{0}^{T}\left\|K_{H}^{-1}\left(\int_{0} G(Z)\right)(t)\right\|^{2} d t\right] \leq c \mathbb{E} \exp \left[\hat{k} c_{T}|\tilde{Z}|_{C([0, T], E)}^{2}\right]
$$

for some $c>0$. Substituting $v=\frac{r}{s}$ in the integral with respect to $r$ on the right-hand side of (3.14), it easily follows that $c_{T} \downarrow 0$ as $T \downarrow 0$. Since $\tilde{Z}$ is a $C([0, T], E)$-valued Gaussian random variable, it follows that

$$
\mathbb{E} \exp \left[\hat{k} c_{T}|\tilde{Z}|_{C([0, T], E)}^{2}\right]<\infty
$$

is satisfied for $T>0$ sufficiently small by the Fernique inequality. Clearly, (3.16) is the Novikov condition [19] which implies the equality (3.12) for $T>0$ sufficiently small. For arbitrary $T>0$, a simple iteration verifies the result, that is,

$$
\mathbb{E} \exp \left[\hat{k} \int_{T_{m-1}}^{T_{m}}\left\|K_{H}^{-1}\left(\int_{0}^{.} G(Z)\right)(t)\right\|^{2} d t\right]<\infty
$$

for a sufficiently fine partition $0=T_{0}<T_{1}<\cdots<T_{n}=T$. Using a downward induction procedure from the well-known proofs of the martingale property for the Radon-Nikodým derivative in (3.12) for an arbitrary $T>0$ (see, e.g., [20, Example 6.2.3]), the verification of the equality in (3.12) is obtained.

Now, uniqueness of the weak solution is verified. Uniqueness in law can be proved in a standard way by removing the term $F$ in (3.4) by absolute continuity of measures, which is a suitable inverse of the above construction of a weak solution.

Let $(\tilde{X}(t), t \in[0, T])$ be a solution to the equation

$$
\tilde{X}(t)=S(t) x_{0}+\int_{0}^{t} S(t-r) F(X(r)) d r+\tilde{Z}(t)
$$

Copyright $@$ by SIAM. Unauthorized reproduction of this article is prohibited. 
where $\tilde{Z}(t)=\int_{0}^{t} S(t-r) \Phi d B(r)$ and $(B(t), t \in[0, T])$ is some standard cylindrical fractional Brownian motion on a probability space $(\tilde{\Omega}, \tilde{\mathcal{F}}, \tilde{\mathbb{P}})$.

The process $(\tilde{X}(t), t \in[0, T])$ is defined on the same probability space as $(B(t), t \in$ $[0, T])$. Let $(W(t), t \in[0, T])$ be the Wiener process associated with $(B(t), t \in[0, T])$ by (2.18). It suffices to show that

$$
\begin{aligned}
& \exp [\tilde{\rho}(\tilde{X})] \\
& :=\exp \left[-\int_{0}^{T}\left\langle\mathbb{K}_{H}^{-1}\left(\int_{0} G(\tilde{X})\right)(t), d W(t)\right\rangle-\frac{1}{2} \int_{0}^{T}\left\|\mathbb{K}_{H}^{-1}\left(\int_{0} G(\tilde{X})\right)(t)\right\|^{2} d t\right]
\end{aligned}
$$

is a Radon-Nikodým derivative on $(\tilde{\Omega}, \tilde{\mathcal{F}}, \tilde{\mathbb{P}})$, so $\tilde{\mathbb{P}}$ is the measure for a fractional Ornstein-Uhlenbeck process and uniqueness in law follows. Thus it is necessary to show that

$$
\int_{0}^{\cdot} G(\tilde{X}(s)) d s \in I_{0+}^{H+\frac{1}{2}}\left(L^{2}([0, T], V)\right)
$$

and

$$
\tilde{\mathbb{E}} \exp [\tilde{\rho}(\tilde{X})]=1,
$$

where $\tilde{E}$ is integration with respect to $\tilde{\mathbb{P}}$. The verifications of (3.20) and (3.21) are analogous to the verifications of (3.11) and (3.12), respectively. However, since $\tilde{X}$ is not a Gaussian process, the Fernique inequality cannot be used directly. Initially, it is verified that there is a $c>0$ such that

$$
|\tilde{X}|_{C([0, T], E)} \leq c\left(1+\left\|X_{0}\right\|_{E}+|\tilde{Z}|_{C([0, T], E)}\right),
$$

where $\tilde{Z}$ is the stochastic process described in (H2). Let

$$
\begin{aligned}
u(t) & =\tilde{X}(t)-\tilde{Z}(t) \\
& =S(t) X_{0}+\int_{0}^{t} S(t-r) F(u(r)+\tilde{Z}(r)) d r .
\end{aligned}
$$

Thus

$$
\|u(t)\|_{E} \leq c_{1}\left\|X_{0}\right\|+c_{2} \int_{0}^{t}\left(1+\|u(r)\|_{E}+\|\tilde{Z}(r)\|_{E}\right) d r
$$

for some positive constants $c_{1}$ and $c_{2}$. By the Gronwall lemma it follows that

$$
\|u(t)\|_{E} \leq c_{1}\left(1+\left\|X_{0}\right\|_{E}+|\tilde{Z}|_{C([0, T], E)}\right)
$$

for $t \in[0, T]$, so the inequality (3.22) is verified. The exponential that usually occurs in the Gronwall inequality is bounded by $e^{c_{2} T}$. Making the analogous computations in (3.14), it follows that

$$
\left|\mathbb{K}_{H}^{-1}\left(\int_{0} G(Z)\right)(s)\right|_{L^{2}([0, T], V)}^{2} \leq c_{T}\left(1+|X|_{C([0, T], E)}^{2}\right) \leq \tilde{c}_{T}\left(1+|\tilde{Z}|_{C([0, T], E)}^{2}\right)
$$


where $\tilde{c}_{T} \downarrow 0$ as $T \downarrow 0$, so (3.20) is satisfied. Thus the method in (3.15)-(3.17) can be used to verify (3.21).

The random variable $\exp (\tilde{\rho}(\tilde{X}))$ in $(3.19)$ is a Radon-Nikodým derivative and it defines a probability measure $\mathbb{Q}$ on $\tilde{\Omega}$. By this Girsanov-type theorem the process defined by $\tilde{B}(t)=B(t)+\int_{0}^{t} u(s) d s$, where $u(s)=G(\tilde{X}(s))$, is a standard cylindrical fractional Brownian motion with respect to the measure $\mathbb{Q}$. Let $(\tilde{W}(t), t \in[0, T])$ be the Wiener process associated with $(\tilde{B}(t), t \in[0, T])$. Let $U(s)=\mathbb{K}_{H}^{-1}\left(\int_{0}^{*} G(\tilde{X})\right)(s)$ for $s \in[0, T]$ and let $\mathbb{E}$ and $\mathbb{E}_{\mathbb{Q}}$ denote the expectations with respect to the measures $\tilde{\mathbb{P}}$ and $\mathbb{Q}$, respectively. For a bounded measurable function $\Psi$ on $C([0, T], V)$ it follows that

$$
\begin{aligned}
\mathbb{E}[\Psi(\tilde{X})]= & \int_{\tilde{\Omega}} \Psi \frac{d \tilde{\mathbb{P}}}{d \mathbb{Q}} d \mathbb{Q}=\mathbb{E}_{\mathbb{Q}}[\Psi(\tilde{X}) \exp (-\tilde{\rho}(\tilde{X}))] \\
= & \mathbb{E}_{\mathbb{Q}}\left[\Psi(\tilde{X}) \exp \left(\int_{0}^{T}\langle U(r), d \tilde{W}(r)\rangle-\frac{1}{2} \int_{0}^{T}\|U(r)\|^{2} d r\right)\right] \\
= & \mathbb{E}_{\mathbb{Q}}\left[\Psi ( S ( \cdot ) X _ { 0 } + \int _ { 0 } ^ { \cdot } S ( \cdot - r ) d \tilde { B } ( r ) ) \operatorname { e x p } \left(\int _ { 0 } ^ { T } \left\langle\mathbb { K } _ { H } ^ { - 1 } \left(\int _ { 0 } G \left(S(\cdot) X_{0}\right.\right.\right.\right.\right. \\
& \left.\left.\left.\quad+\int_{0}^{\cdot} S(\cdot-r) \Phi d \tilde{B}(r)\right)\right)(s), d \tilde{W}(s)\right\rangle \\
& \left.\left.-\frac{1}{2} \int_{0}^{T}\left\|\mathbb{K}_{H}^{-1}\left(\int_{0}^{\cdot} G\left(S(\cdot) X_{0}+\int_{0}^{\cdot} S(\cdot-r) \Phi d \tilde{B}(r)\right)\right)(s)\right\|^{2} d s\right)\right] .
\end{aligned}
$$

Since the processes $\tilde{W}$ and $\tilde{B}$ are standard cylindrical Brownian motions and standard cylindrical fractional Brownian motions, respectively, the final expectation on the right-hand side above does not depend on the realization of $\tilde{X}$, so the uniqueness in law is verified.

Now the existence and the uniqueness of a weak solution of (3.4) is verified for $H \in(1 / 2,1)$.

THEOREM 3.4. If $H \in(1 / 2,1)$, (H1)-(H3) are satisfied, and

$$
\|G(x)-G(y)\| \leq k_{G}\|x-y\|^{\gamma}
$$

for all $x, y \in E$, some $\gamma \in(0,1], k_{G}>0$, and $\tilde{Z} \in C^{\beta}([0, T], V)$ for some $\beta$ satisfying

$$
\beta>\frac{H-\frac{1}{2}}{\gamma},
$$

where $\tilde{Z}$ is the stochastic convolution process in (H2), then (3.4) has a weak solution. If, additionally, (3.10) is satisfied, then the weak solution is unique.

Proof. Initially, the existence of a solution is verified as in the proof of Theorem 3.3. It is shown that

$$
\int_{0}^{.} G(Z(s)) d s \in I_{0+}^{H+\frac{1}{2}}\left(L^{2}([0, T], V)\right) \text { a.s. }
$$

and

$$
\mathbb{E} \exp [\rho(Z)]=1
$$

Copyright $@$ by SIAM. Unauthorized reproduction of this article is prohibited. 
where $\rho$ is given by (3.13). By (2.8) it follows that

$$
\begin{aligned}
&\left|\mathbb{K}_{H}^{-1}\left(\int_{0} G(Z)\right)\right|_{L^{2}([0, T], V)}^{2} \\
&=c_{H}^{-2}\left|u_{H-\frac{1}{2}} D_{0+}^{H-\frac{1}{2}}\left(u_{\frac{1}{2}-H} G(Z)\right)\right|_{L^{2}([0, T], V)}^{2} \\
&=c_{H}^{-2} \int_{0}^{T} \| \frac{s^{H-\frac{1}{2}}}{\Gamma\left(\frac{3}{2}-H\right)}\left(\frac{s^{\frac{1}{2}-H} G(Z(s))}{s^{H-\frac{1}{2}}}\right) \\
& \\
& \quad+\left(H-\frac{1}{2}\right) \int_{0}^{s} \frac{s^{\frac{1}{2}-H} G(Z(s))-r^{\frac{1}{2}-H} G(Z(r))}{(s-r)^{H+\frac{1}{2}}} d r \|^{2} d s \\
& \leq c \int_{0}^{T}\left(s^{\frac{1}{2}-H}\|G(Z(s))\|+s^{H-\frac{1}{2}} \int_{0}^{s} \frac{s^{\frac{1}{2}-H}-r^{\frac{1}{2}-H}}{(s-r)^{H+\frac{1}{2}}}\|G(Z(r))\| d r\right. \\
&\left.+\int_{0}^{s} \frac{\|G(Z(s))-G(Z(r))\|}{(s-r)^{H+\frac{1}{2}}} d r\right)^{2} d s .
\end{aligned}
$$

Using (3.9) and (3.26), the analyticity of the semigroup $S(\cdot)$ on $V$, and the inequality

$$
\int_{0}^{s} \frac{s^{\frac{1}{2}-H}-r^{\frac{1}{2}-H}}{(s-r)^{H+\frac{1}{2}}} d r \leq c s^{1-2 H}
$$

where $c$ is a generic constant, it follows that

$$
\begin{aligned}
& \left|\mathbb{K}_{H}^{-1}\left(\int_{0}^{.} G(Z)\right)\right|_{L^{2}([0, T], V)} \\
& \quad \leq c_{T}\left(1+\left\|X_{0}\right\|_{E}^{2}+|\tilde{Z}|_{C([0, T], E)}^{2}\right) \\
& \quad+c_{T} \int_{0}^{T}\left[\left(\int_{0}^{s} \frac{\left\|S(s) X_{0}-S(r) X_{0}\right\|^{\gamma}}{(s-r)^{H+\frac{1}{2}}} d r\right)^{2}+\left(\int_{0}^{s} \frac{\|\tilde{Z}(s)-\tilde{Z}(r)\|^{\gamma}}{(s-r)^{H+\frac{1}{2}}} d r\right)^{2}\right] d s \\
& \quad \leq c_{T}\left(1+\left\|X_{0}\right\|_{E}^{2}+|\tilde{Z}|_{C([0, T], E)}^{2}\right)+c_{T} \int_{0}^{T}\left(\left\|X_{0}\right\|^{\gamma} \int_{0}^{s} \frac{(s-r)^{\gamma \lambda}}{r^{\gamma \lambda}(s-r)^{H+\frac{1}{2}}} d r\right)^{2} \\
& \quad+c_{T} \int_{0}^{T}|\tilde{Z}|_{C^{\beta}([0, T], V)}^{2}\left(\int_{0}^{s} \frac{(s-r)^{\gamma \beta}}{(s-r)^{H+\frac{1}{2}}} d r\right)^{2} d s,
\end{aligned}
$$

where $\lambda>0$ satisfies $\gamma \lambda<1$ and $H+1 / 2-\gamma \lambda<1$. The first integral term in the initial inequality in (3.31) is obtained by the analyticity of the semigroup $S($.$) on V$, which implies that

$$
\|(S(s)-S(r)) x\| \leq c(s-r)^{\lambda} r^{\lambda}\|x\|, \quad x \in V, \lambda \geq 0,0<r<s \leq T,
$$

Copyright (c) by SIAM. Unauthorized reproduction of this article is prohibited. 
for some $c=c(\lambda)$. It follows that

$$
\left|\mathbb{K}_{H}^{-1}\left(\int_{0} G(Z)\right)\right|_{L^{2}([0, T], V)}^{2} \leq c_{T}\left(1+\left\|X_{0}\right\|_{E}^{2}+\|\tilde{Z}\|_{C([0, T], E)}^{2}+|\tilde{Z}|_{C^{\beta}([0, T], V)}^{2}\right),
$$

where $c_{T} \downarrow 0$ as $T \downarrow 0$, so (3.28) is verified and by the Fernique inequality (3.29) is also verified.

Now the uniqueness of the weak solution is verified. Let $(\tilde{X}(t), t \in[0, T])$ be the solution to $(3.4)$ on a probability space $(\tilde{\Omega}, \tilde{\mathcal{F}} . \tilde{\mathbb{P}})$. As in the proof of Theorem 3.3 , it is shown that

$$
\int_{0}^{\cdot} G(\tilde{X}) d s \in I_{0+}^{H+\frac{1}{2}}\left(L^{2}([0, T], V)\right) \quad \text { a.s. }
$$

and

$$
\tilde{\mathbb{E}} \exp [\tilde{\rho}(\tilde{X})]=1
$$

where $\tilde{\rho}$ is given by (3.13). It is necessary to obtain inequality (3.22), used in the proof of Theorem 3.3. Inequality (3.22) is verified by verifying the inequality

$$
\|\hat{X}\|_{C^{\beta}([0, T], V)} \leq L\left(1+\left\|X_{0}\right\|_{E}+|\tilde{Z}|_{C([0, T], E)}+|\tilde{Z}|_{C^{\beta}([0, T], V)}\right)
$$

where $\hat{X}(t)=\tilde{X}(t)-S(t) X_{0}$ and $L>0$. Let $w(t)=\tilde{X}(t)-S(t) X_{0}-\tilde{Z}(t)$ for $t \geq 0$. The process $w$ satisfies

$$
w(t)=\int_{0}^{t} S(t-r) \psi(r) d r
$$

for $t \in[0, T]$, where

$$
\psi(t)=F\left(w(t)+S(t) X_{0}+\tilde{Z}(t)\right)
$$

By inequalities (3.10) and (3.35) it follows that $\psi \in L^{\infty}([0, T], V)$ a.s. $\mathbb{P}$. Since the semigroup $S(\cdot)$ is analytic on $V, w$ is $\alpha$-Hölder continuous for each $\alpha \in(0,1)$, and using the method of proof of [29, Theorem 4.3.1] there are constants $c_{i}>0$ for $i=1,2$ such that

$$
|w|_{C^{\beta}([0, T], V)} \leq c_{1}|\psi|_{L^{\infty}([0, T], V)} \leq c_{2}\left(|w|_{L^{\infty}([0, T], E)}+\left\|X_{0}\right\|_{E}+|\tilde{Z}|_{C([0, T], E)}\right) .
$$

Thus

$$
\begin{aligned}
|\tilde{X}|_{C^{\beta}([0, T], V)} & \leq|w|_{C^{\beta}([0, T], V)}+|\tilde{Z}|_{C^{\beta}([0, T], V)} \\
& \leq c_{2}\left(|w|_{L^{\infty}([0, T], E)}+\left\|X_{0}\right\|_{E}+|\tilde{Z}|_{C([0, T], E)}+|\tilde{Z}|_{C^{\beta}([0, T], V)}\right) .
\end{aligned}
$$

Using (3.22) again to bound $|w|_{L^{\infty}([0, T], E)}$, inequality (3.35) follows.

Copyright (C) by SIAM. Unauthorized reproduction of this article is prohibited. 
Now, using the methods for inequalities (3.30)-(3.32), where $Z(t)$ is replaced by $\tilde{X}(t)=S(t) X_{0}+\hat{X}(t)$, it follows that

$$
\left|\mathbb{K}_{H}^{-1}\left(\int_{0}^{\cdot} G(Z)\right)\right|_{L^{2}([0, T], V)}^{2} \leq c_{T}\left(1+\left\|X_{0}\right\|_{E}^{2}+|\tilde{X}|_{C([0, T], V)}^{2}+|\tilde{X}|_{C^{\beta}([0, T], V)}^{2}\right),
$$

where $c_{T} \downarrow 0$ as $T \downarrow 0$, which by (3.22) and (3.35) verifies (3.33). Equality (3.34) is obtained from (3.39) by the Fernique inequality as in the proof of Theorem 3.3.

Remark 3.5. The proofs of Theorems 3.3 and 3.4 have verified, in addition to weak existence and uniqueness of a solution to (3.4), the mutual absolute continuity (equivalence) of the probability laws of the solution to (3.4) and the solution of (3.4) with $F \equiv 0$ (the fractional Ornstein-Uhlenbeck process) in the path space.

The next objective is to relax the linear growth conditions (3.9) and (3.10) and the Hölder continuity (3.26). The linear growth condition is replaced by a dissipativity condition of the drift term of (3.4), but some other conditions are also imposed so that there is existence and (strong) uniqueness of a mild solution. The main contribution of the following two theorems is a mutual absolute continuity of the probability laws of the solutions of (3.4) with a nonzero $F$ and (3.4) with $F \equiv 0$.

Initially, the case $H \in(0,1 / 2)$ is considered.

Theorem 3.6. Let $H \in(0,1 / 2)$ and let (H1) and (H2) be satisfied. Let $\Phi \in$ $\mathcal{L}(V)$ be injective, let $\Phi^{-1} \in \mathcal{L}(E, V)$, and let $\left(\left.S(t)\right|_{E}, t \geq 0\right)$ be a strongly continuous semigroup on $E$ such that

$$
\left.|S(t)|_{E}\right|_{\mathcal{L}(E)} \leq e^{\tilde{w} t}
$$

for $t \geq 0$ and some $\tilde{w} \in \mathbb{R}$. Let $F: E \rightarrow E$ be continuous and satisfy

$$
\|F(x)\|_{E} \leq k_{1}\left(1+\|x\|_{E}^{\rho}\right)
$$

for $x \in E$ for some $k_{1} \geq 0$ and $\rho \geq 1$, and for each pair $x, y \in E$, there is a $z^{*} \in \partial\|x-y\|_{E}$ where $\partial\|z\|_{E}$ is the subdifferential of the norm $\|\cdot\|_{E}$ at $z \in E$ such that

$$
\left\langle F(x)-F(y), z^{*}\right\rangle_{E, E^{*}} \leq k_{2}\|x-y\|_{E}
$$

for some $k_{2} \in \mathbb{R}$; that is, $F-k_{2} I$ is dissipative on $E$. Then there is one and only one mild solution of (3.4), and its probability law on the Borel $\sigma$-algebra of $\check{\Omega}=$ $C([0, T], E)$ is mutually absolutely continuous with respect to the probability law of the fractional Ornstein-Uhlenbeck process (3.24) on $\Omega$.

Proof. Let $\left(F_{\lambda}, \lambda>0\right)$ be a family of Lipschitz continuous functions from $E$ to $E$ such that each $F_{\lambda}$ satisfies inequalities (3.41) and (3.42) for $F$ with the same constants $\rho, k_{1}, k_{2}$. It is shown that there is a $\bar{k}>0$ depending only on $\tilde{w}, k_{1}$, and $k_{2}$ such that

$$
\left\|v_{\lambda}(t)\right\|_{E} \leq \bar{k}\left(1+\left\|X_{0}\right\|_{E}+\|\phi\|_{C([0, T], E)}^{\rho}\right)
$$

for $t \in[0, T]$ is satisfied for each $\lambda>0$ and $\phi \in C([0, T], E)$, where $v_{\lambda}$ is a solution of the equation

$$
v_{\lambda}(t)=S(t) X_{0}+\int_{0}^{t} S(t-r) F_{\lambda}\left(v_{\lambda}(r)+\phi(r)\right) d r
$$

for $t \in[0, T]$. 
To verify inequality (3.43), it can be assumed by translation that $k_{2}=0$ in (3.42) (replace $F_{\lambda}$ and $A$ by $F_{\lambda}-k_{2} I$ and $A+k_{2} I$, respectively). Thus $F_{\lambda}$ is dissipative on $E$ for each $\lambda>0$ and by the assumptions

$$
\left\langle A_{E} z, z^{*}\right\rangle_{E, E^{*}} \leq \tilde{w}\|z\|_{E}^{2}
$$

for each $z \in \operatorname{Dom}\left(A_{E}\right)$ and $z^{*} \in \partial\|z\|_{E}$, where $A_{E}$ is the restriction of $A$ to $E$ that generates the semigroup $\left.S(\cdot)\right|_{E}$. For each pair $x, y \in \operatorname{Dom}\left(A_{E}\right)$ and $\lambda>0$, there is a $z_{\lambda}^{*} \in \partial\|x-y\|_{E}$ such that

$$
\left\langle A_{E}(x-y)+F_{\lambda}(x)-F_{\lambda}(y), z_{\lambda}^{*}\right\rangle_{E, E^{*}} \leq \tilde{w}\|x-y\|_{E} .
$$

By [5, Proposition 5.5.6], there is a sequence $\left(v_{\lambda}^{n}, n \in \mathbb{N}\right)$ such that $v_{\lambda}^{n} \in C^{1}([0, T], E) \cap$ $C\left([0, T], \operatorname{Dom}\left(A_{E}\right)\right)$ such that $v_{\lambda}^{n} \rightarrow v_{\lambda}$ and $\delta_{\lambda}^{n}=\frac{d}{d t} v_{\lambda}^{n}-A_{E} v_{\lambda}^{n}-F_{\lambda}\left(v_{\lambda}^{n}+\phi\right) \rightarrow 0$ in $C([0, T], E)$ as $n \rightarrow \infty$. It follows that

$$
\begin{aligned}
\frac{d^{-}}{d t}\left\|v_{\lambda}^{n}(t)\right\|_{E} \leq & \left\langle A_{E} v_{\lambda}^{n}(t)+F_{\lambda}\left(v_{\lambda}^{n}(t)+\phi(t)\right),\left(v_{\lambda}^{n}(t)\right)^{*}\right\rangle_{E, E^{*}}+\left\|\delta_{\lambda}^{n}(t)\right\|_{E} \\
= & \left\langle A_{E} v_{\lambda}^{n}(t)+F_{\lambda}\left(v_{\lambda}^{n}(t)+\phi(t)\right)-F_{\lambda}(\phi(t)),\left(v_{\lambda}^{n}(t)\right)^{*}\right\rangle \\
& \quad+\left\langle F_{\lambda}(\phi(t)),\left(v_{\lambda}^{n}(t)\right)^{*}\right\rangle_{E, E^{*}}+\left\|\delta_{x}^{n}(t)\right\|_{E} \\
& \leq \bar{w}\left\|v_{\lambda}^{n}(t)\right\|_{E}+k_{2}\left(1+|\phi|_{C([0, T], E)}^{\rho}+\left\|\delta_{\lambda}^{n}(t)\right\|_{E}\right)
\end{aligned}
$$

for $t \in[0, T]$. Using the Gronwall lemma, and letting $n \rightarrow \infty$, verifies inequality (3.43).

The mild solution to (3.4) can be expressed as $X(t)=v(t)+\tilde{Z}(t)$, where $v$ satisfies the equation

$$
v(t)=S(t) X_{0}+\int_{0}^{t} S(t-r) F(v(r)+\tilde{Z}(r)) d r
$$

for $t \in[0, T]$. Thus the existence and the uniqueness of a mild solution follows from the corresponding pathwise deterministic result (cf. [5, Proposition 5.5.6]).

The equivalence of the probability laws is shown by application of Theorem 3.1. As in the proof of Theorem 3.3, it suffices to show that

$$
\int_{0}^{\cdot} G(Z(s)) d s \in I_{0+}^{H+\frac{1}{2}}\left(L^{2}([0, T], V)\right)
$$

and

$$
\mathbb{E} \exp [\rho(Z)]=1
$$

where $\rho$ is given by (3.13). While $G$ is not assumed to have at most linear growth as in Theorem 3.3, there is the growth condition

$$
\|G(x)\| \leq \hat{k}\left(1+\|x\|_{E}^{\rho}\right)
$$

Copyright $@$ by SIAM. Unauthorized reproduction of this article is prohibited. 
for all $x \in E$ and a constant $\hat{k}$. Proceeding as in (3.14), it follows that

$$
\begin{aligned}
& \left|\mathbb{K}_{H}^{-1}\left(\int_{0}^{\cdot} G(Z)\right)\right|_{L^{2}([0, t], V)}^{2} \\
& \quad \leq c_{1} \int_{0}^{T}\left(s^{H-\frac{1}{2}}\left\|\int_{0}^{s} r^{\frac{1}{2}-H}(s-r)^{-\frac{1}{2}-H} G(Z(r)) d r\right\|\right)^{2} d s \\
& \quad \leq c_{2}\left(1+|\tilde{Z}|_{C([0, T], E)}^{\rho}+\sup _{t \in[0, T]}\left\|S(t) X_{0}\right\|_{E}^{\rho}\right) \int_{0}^{T} s^{2 H-1} \\
& \quad \cdot\left(\int_{0}^{s} r^{\frac{1}{2}-H}(s-r)^{-\frac{1}{2}-H} d r\right)^{2} d s \\
& \quad \leq c_{3}\left(1+\left\|X_{0}\right\|_{E}^{2 \rho}+|\tilde{Z}|_{C([0, T], E)}^{2 \rho}\right)
\end{aligned}
$$

for suitable constants $c_{1}, c_{2}, c_{3}$. This inequality verifies (3.48). To verify equality (3.49), it suffices to assume that $F$ is dissipative (that is, $k_{2}=0$ in (3.42)). Recall that $m$-dissipative mapping $F$ is defined as a dissipative mapping satisfying Range $(I-$ $\lambda F)=E$ for each $\lambda>0$; cf. [22]. Since $F$ is continuous, it is $m$-dissipative (cf. [23]), so the family $\left(F_{\lambda}, \lambda>0\right)$ of Yosida approximations of $\mathrm{F}$ is defined as

$$
F_{\lambda}(x)=F\left(R_{\lambda}(x)\right)=\frac{1}{\lambda}\left(R_{\lambda}(x)-x\right)
$$

for $x \in E$, where

$$
R_{\lambda}(x)=(I-\lambda F)^{-1}(x) .
$$

It is well known that $F_{\lambda}: E \rightarrow E$ for $\lambda>0$ is Lipschitz continuous, so by Theorem 3.3, there is the equality

$$
\mathbb{E} \exp \left[\rho_{\lambda}(Z)\right]=1
$$

for $\lambda>0$, where

$$
\rho_{\lambda}(Z)=\int_{0}^{T}\left\langle\mathbb{K}_{H}^{-1}\left(\int_{0} G_{\lambda}(Z)\right)(t), d W(t)\right\rangle-\frac{1}{2} \int_{0}^{T}\left\|\mathbb{K}_{H}^{-1}\left(\int_{0} G_{\lambda}(Z)\right)(t)\right\|^{2} d t
$$

and $G_{\lambda}:=\Phi^{-1} F_{\lambda}$. As in (3.51), it follows that

$$
\begin{aligned}
& \mathbb{E}\left|\mathbb{K}_{H}^{-1}\left(\int_{0}^{\cdot}\left(G_{\lambda}(Z)-G(Z)\right)\right)\right|_{L^{2}([0, T], V)} \\
& \leq c_{T} \mathbb{E} \int_{0}^{T}\left(s^{H-\frac{1}{2}} \int_{0}^{s} r^{\frac{1}{2}-H}(s-r)^{-\frac{1}{2}-H}\left\|G_{\lambda}(Z(r))-G(Z(r))\right\| d r\right)^{2} d s .
\end{aligned}
$$

By some well-known properties of the Yosida approximations and for $x \in E$,

$$
\left\|G_{\lambda}(x)-G(x)\right\| \leq\left|\Phi^{-1}\right|_{\mathcal{L}(E, V)}\left\|F_{\lambda}(x)-F(x)\right\|,
$$

Copyright $@$ ( ) by SIAM. Unauthorized reproduction of this article is prohibited. 
it follows that $F_{\lambda} \rightarrow F$ as $\lambda \rightarrow 0$ and the right-hand side of (3.57) tends to zero as $\lambda \downarrow 0$, and

$$
\begin{aligned}
\left\|G_{\lambda}(x)\right\| & \leq\left|\Phi^{-1}\right|_{\mathcal{L}(E, V)}\left\|F_{\lambda}(x)\right\|_{E} \\
& \leq\left|\Phi^{-1}\right|_{\mathcal{L}(E, V)}\|F(x)\|_{E} \\
& \leq\left|\Phi^{-1}\right|_{\mathcal{L}(E, V)} k_{1}\left(1+\|x\|_{E}^{\rho}\right),
\end{aligned}
$$

so the right-hand side of (3.56) tends to zero as $\lambda \downarrow 0$. For a sequence $\left(\lambda_{n}, n \in \mathbb{N}\right)$ that decreases to zero, it follows that

$$
\lim _{n \rightarrow \infty} \exp \left[\rho_{\lambda_{n}}(Z)\right]=\exp [\rho(Z)] \quad \text { a.s. } \mathbb{P} \text {. }
$$

To obtain equality (3.54) from equality (3.59) for $\lambda_{n}, n \in \mathbb{N}$, it is necessary and sufficient to show that the sequence $\left(\exp \left[\rho_{\lambda_{n}}(Z)\right], n \in \mathbb{N}\right)$ is uniformly integrable. A sufficient condition for this uniform integrability is to verify that

$$
\sup _{n} \mathbb{E}\left[\left(\exp \left[\rho_{\lambda_{n}}(Z)\right]\right)\left|\log \left(\exp \left[\rho_{\lambda_{n}}(Z)\right]\right)\right|\right]=\sup _{n} \mathbb{E}\left[\left(\exp \left[\rho_{\lambda_{n}}(Z)\right]\right) \rho_{\lambda_{n}}(Z)\right]<\infty .
$$

By Theorem 3.3,

$$
\mathbb{E}\left[\rho_{\lambda_{n}}(Z) \exp \left[\rho_{\lambda_{n}}(Z)\right]\right] \leq \tilde{\mathbb{E}}_{\lambda_{n}}\left[2 \int_{0}^{T}\left\|\mathbb{K}_{H}^{-1}\left(\int_{0} G_{\lambda_{n}}(Z)\right)(t)\right\|^{2} d t\right],
$$

where $\tilde{\mathbb{E}}_{\lambda_{n}}$ is the expectation with respect to $\tilde{\mathbb{P}}_{\lambda_{n}}$ and

$$
\frac{d \tilde{\mathbb{P}}_{\lambda_{n}}}{d \mathbb{P}}=\exp \left[\rho_{\lambda_{n}}(Z)\right]
$$

and $Z(\cdot)$ satisfies (2.28). On the probability space with the measure $\mathbb{P}_{\lambda_{n}}, Z(\cdot)$ satisfies the following semilinear equation, where $B(\cdot)$ is a fractional Brownian motion with respect to $\mathbb{P}_{\lambda_{n}}$ :

$$
\begin{aligned}
d X_{\lambda_{n}}(t) & =\left(A X(t)+F_{\lambda_{n}}(X(t))\right) d t+\Phi d B(t), \\
X_{\lambda_{n}}(0) & =X_{0} .
\end{aligned}
$$

Since $F_{\lambda_{n}}$ is Lipschitz continuous, there is a unique mild solution on a given probability space, so it suffices to show

$$
\mathbb{E} \int_{0}^{T}\left\|\mathbb{K}_{H}^{-1}\left(\int_{0}^{\cdot} G_{\lambda_{n}}\left(X_{\lambda_{n}}\right)\right)(t)\right\|^{2} d t \leq c
$$

for some $c \in \mathbb{R}_{+}$that does not depend on $\lambda_{n}$. Repeating inequalities (3.51), where $G$ and $Z$ are replaced by $G_{\lambda_{n}}$ and $X_{\lambda_{n}}$, respectively, and using inequality (3.58), it follows that

$$
\int_{0}^{T}\left\|\mathbb{K}_{H}^{-1}\left(\int_{0} G_{\lambda_{n}}\left(X_{\lambda_{n}}\right)\right)(t)\right\|^{2} d t \leq c_{5}\left(1+\left\|X_{0}\right\|_{E}^{2 \rho}+\left|\tilde{X}_{\lambda_{n}}\right|_{C([0, T], E)}^{2 \rho}\right)
$$

Copyright (C) by SIAM. Unauthorized reproduction of this article is prohibited. 
for a constant $c_{5}$ that does not depend on $n \in \mathbb{N}$ where $\tilde{X}_{\lambda_{n}}(t)=X_{\lambda_{n}}(t)-S(t) X_{0}$. By inequality (3.43) there is a constant $c_{6}$ that does not depend on $n$ such that

$$
\mathbb{E} \int_{0}^{T}\left\|\mathbb{K}_{H}^{-1}\left(\int_{0}^{\cdot} G_{\lambda_{n}}\left(X_{\lambda_{n}}\right)\right)(t)\right\|^{2} d t \leq c_{6}\left(1+\left\|X_{0}\right\|_{E}^{2 \rho}+\mathbb{E}|Z|_{C([0, T], E)}^{4 \rho^{2}}\right)=C<\infty .
$$

This inequality verifies (3.60). Thus the sequence $\left(\exp \left[\rho_{\lambda_{n}}(Z)\right], n \in \mathbb{N}\right)$ converges in $L^{1}$ and equality (3.54) is satisfied.

Now the case $H \in(1 / 2,1)$ is considered.

THEOREM 3.7. Let $H \in(1 / 2,1)$ and the other assumptions in Theorem 3.6 be satisfied. Let $\Phi^{-1} \in \mathcal{L}(V), \tilde{Z} \in C^{\beta}([0, T], V)$ for some $\beta \in(0,1)$,

$$
\langle F(x)-F(y), x-y\rangle \leq k_{2}\|x-y\|^{2}
$$

for each pair $x, y \in E$ and $a k_{2} \in \mathbb{R}_{+}$(that is, $F-k_{2} I$ is dissipative on $E$ with respect to the norm on $V$ ) and

$$
\|F(x)-F(y)\| \leq k_{3}\left(1+\|x\|_{E}^{q}+\|y\|_{E}^{q}\right)\|x-y\|^{\gamma}
$$

for each $x, y \in E$, with some $k_{3}>0, q \geq 1$, and $\gamma \in(0,1]$ such that

$$
\gamma \beta>H-\frac{1}{2} \text {. }
$$

Then there is one and only one mild solution to (3.4), and its probability law is mutually absolutely continuous with respect to the probability law of the fractional Ornstein-Uhlenbeck process (2.28) on $\check{\Omega}$.

Proof. As in the proof of Theorem 3.6, it is shown that

$$
\int_{0}^{\cdot} G(Z(s)) d s \in I_{0+}^{H+\frac{1}{2}}\left(L^{2}([0, T], V)\right)
$$

and

$$
\mathbb{E} \exp [\rho(Z)]=1 .
$$

The methods to verify (3.69) and (3.70) are similar to those used in the proof of Theorem 3.6, but now the operator $\mathbb{K}_{H}^{-1}$ has a different form. Using inequality (3.41) and the Hölder continuity condition (3.67), it follows that

$$
\begin{aligned}
& \left|\mathbb{K}_{H}^{-1}\left(\int_{0} G(Z)\right)\right|_{L^{2}([0, T], V)}^{2} \\
& \quad \leq c_{1} \int_{0}^{T}\left(s^{\frac{1}{2}-H}\|G(Z(s))\|+s^{H-\frac{1}{2}} \int_{0}^{s} \frac{s^{\frac{1}{2}-H}-r^{\frac{1}{2}-H}}{(s-r)^{H+\frac{1}{2}}}\|G(Z(r))\| d r\right. \\
& \left.\quad+\int_{0}^{s} \frac{\|G(Z(s))-G(Z(r))\|}{(s-r)^{H+\frac{1}{2}}} d r\right)^{2} d s \\
& \quad \leq c_{2}\left[1+|Z|_{C([0, T], E)}^{2 \rho}+c_{3}\left(1+|Z|_{C([0, T], E)}^{2 q}\right)\right. \\
& \left.\quad \cdot \int_{0}^{T}\left(\int_{0}^{s} \frac{\left\|S(s) X_{0}-S(r) X_{0}\right\|^{\gamma}+\|\tilde{Z}(s)-\tilde{Z}(r)\|^{\gamma}}{(s-r)^{H+\frac{1}{2}}} d r\right)^{2} d s\right]
\end{aligned}
$$

Copyright $@$ by SIAM. Unauthorized reproduction of this article is prohibited. 
for some constants $c_{1}, c_{2}, c_{3}$. By the analyticity of the semigroup $S(\cdot)$ on $V$, it follows that

$$
\begin{aligned}
& \left|\mathbb{K}_{H}^{-1}\left(\int_{0}^{\cdot} G(Z)\right)\right|_{L^{2}([0, T], V)}^{2} \\
& \quad \leq c_{4}\left[1+\left\|X_{0}\right\|_{E}^{2 \rho}\right. \\
& \left.\quad+\left(\left\|X_{0}\right\|_{E}^{2 q}+|Z|_{C([0, T], V)}^{2 q}+1\right)\left(\left\|X_{0}\right\|_{E}^{2 \gamma}+|Z|_{C^{\beta}([0, T], V)}^{2 \gamma}\right)\right] \\
& \quad \leq c_{5}\left(1+\left\|X_{0}\right\|_{E}^{m}+|Z|_{C([0, T], V)}^{m}+|Z|_{C^{\beta}([0, T], V)}^{m}\right)
\end{aligned}
$$

for some constants $c_{4}$ and $c_{5}$ and $m$ sufficiently large. Thus (3.69) is verified. To verify equality (3.70) consider the family of Yosida approximations $\left(F_{\lambda}, \lambda>0\right)$ of $F$ as in the proof of Theorem 3.6. By the dissipativity of $F$ in the norm on $V, F_{\lambda}: V \rightarrow V$ is Lipschitz continuous for each $\lambda>0$ and has at most polynomial growth, so $F_{\lambda}$ satisfies the assumptions of Theorem 3.4 so that

$$
\mathbb{E} \exp \left[\rho_{\lambda}(Z)\right]=1,
$$

where $\rho_{\lambda}$ is given by (3.55). By the method used to obtain inequality (3.71), it follows that

$$
\begin{aligned}
& \mathbb{E}\left|\mathbb{K}_{H}^{-1}\left(\int_{0}^{\cdot}\left(G_{\lambda}(Z)-G(Z)\right)\right)\right|_{L^{2}([0, T], V)}^{2} \\
& \quad \leq c_{6} \mathbb{E} \int_{0}^{T}\left[s^{\frac{1}{2}-H}\left\|G_{\lambda}(Z(s))-G(Z(s))\right\|\right. \\
& +s^{H-\frac{1}{2}} \int_{0}^{s} \frac{s^{\frac{1}{2}-H}-r^{\frac{1}{2}-H}}{(s-r)^{H+\frac{1}{2}}}\left\|G_{\lambda}(Z(r))-G(Z(r))\right\| d r \\
& \left.\quad+\int_{0}^{s} \frac{\left\|G_{\lambda}(Z(s))-G(Z(s))-G_{\lambda}(Z(r))+G(Z(r))\right\|}{(s-r)^{H+\frac{1}{2}}} d r\right]^{2} d s .
\end{aligned}
$$

By inequalities (3.57) and (3.58), it follows that $\left\|G_{\lambda}(x)-G(x)\right\| \rightarrow 0$ as $\lambda \downarrow 0$ for each $x \in E$ and the family $\left(G_{\lambda}, \lambda>0\right)$ satisfies the growth condition

$$
\left\|G_{\lambda}(x)\right\| \leq c_{7}\left(1+\|x\|_{E}^{\rho}\right)
$$

for $x \in E$ and some $c_{7}>0$. From the $V$-dissipativity of $F$, it follows by [5, Proposition 5.5.3] that

$$
\left\|R_{\lambda}(x)-R_{\lambda}(y)\right\| \leq\|x-y\|
$$

for $x, y \in E$, so that

$$
\begin{aligned}
\left\|F_{\lambda}(x)-F_{\lambda}(y)\right\| & =\left\|F\left(R_{\lambda}(x)\right)-F\left(R_{\lambda}(y)\right)\right\| \\
& \leq k_{3}\left(1+\left\|R_{\lambda}(x)\right\|_{E}^{q}+\left\|R_{\lambda}(y)\right\|_{E}^{q}\right)\|x-y\|^{\gamma}
\end{aligned}
$$

for $x, y \in E$. Since

$$
\left\|R_{\lambda}(x)\right\|_{E} \leq\|x\|_{E}+\lambda\|F(x)\|_{E} \leq c_{8}\left(1+\|x\|_{E}^{\rho}\right)
$$

Copyright (c) by SIAM. Unauthorized reproduction of this article is prohibited. 
for $x \in E, c_{8} \in \mathbb{R}_{+}$, and $\lambda \in(0,1]$, there is the inequality

$$
\left\|F_{\lambda}(x)-F_{\lambda}(y)\right\| \leq c_{9}\left(1+\|x\|_{E}^{m}+\|y\|_{E}^{m}\right)\|x-y\|^{\gamma}
$$

for $x, y \in E, c_{0} \in \mathbb{R}_{+}, m \geq 1$, and $\lambda \in(0,1]$. So $F_{\lambda}$ and $G_{\lambda}$ satisfy inequality (3.67) uniformly in $\lambda \in(0,1]$. Thus the right-hand side of inequality (3.74) tends to zero as $\lambda \downarrow 0$ by the dominated convergence theorem where a majorizing function is provided by the estimates (3.75) and (3.77), whose integrability is shown in (3.71) and (3.72), and there is a decreasing sequence $\left(\lambda_{n}, n \in \mathbb{N}\right)$ whose limit is zero such that

$$
\lim _{n \rightarrow \infty} \exp \left[\rho_{\lambda_{n}}(Z)\right]=\exp [\rho(Z)] \quad \text { a.s. } \mathbb{P} \text {. }
$$

The uniform integrability of the sequence $\left(\exp \left[\rho_{\lambda_{n}}(Z)\right], n \in \mathbb{N}\right)$ is shown by verifying the analogue of (3.60). Equivalently,

$$
\sup _{n} \mathbb{E} \int_{0}^{T}\left\|\mathbb{K}_{H}^{-1}\left(\int_{0}^{\cdot} G_{\lambda_{n}}\left(X_{\lambda_{n}}\right)\right)(t)\right\|^{2} d t \leq c<\infty,
$$

where $X_{\lambda_{n}}(\cdot)$ is the unique mild solution to (3.62). The analogous inequalities (3.71)(3.74) are obtained by replacing $G$ by $G_{\lambda}$ using the polynomial growth bound and the local Hölder continuity that are uniform in $\left(\lambda_{n}, n \in \mathbb{N}\right)$, and $Z(\cdot)$ is replaced by $X_{\lambda_{n}}(\cdot)$. For some constants $c_{10}$ and $m \geq 1$,

$$
\begin{aligned}
& \left|\mathbb{K}_{H}^{-1}\left(\int_{0} G_{\lambda_{n}}\left(X_{\lambda_{n}}\right)\right)\right|_{L^{2}([0, T], V)}^{2} \\
& \quad \leq c_{10}\left(1+\left\|X_{0}\right\|_{E}^{m}+\left|\tilde{X}_{\lambda_{n}}\right|_{C([0, T], E)}^{m}+\left|\tilde{X}_{\lambda_{n}}\right|_{C^{\beta}([0, T], V)}^{m}\right),
\end{aligned}
$$

where $\tilde{X}_{\lambda_{m}}(t)=X_{\lambda_{m}}(t)-S(t) X_{0}$. By inequality (3.43), it follows that

$$
\left|\tilde{X}_{\lambda_{n}}\right|_{C([0, T], E)} \leq c_{11}\left(1+\left\|X_{0}\right\|+|Z|_{C(0, T], E)}^{\rho}\right)
$$

for some $c_{11}>0$. Let $w_{\lambda_{n}}(t)=\tilde{X}_{\lambda_{n}}(t)-\tilde{Z}(t)$ so that

$$
w_{\lambda_{n}}(t)=\int_{0}^{t} S(t-r) F_{\lambda_{n}}\left(w_{\lambda_{n}}(s)+S(s) X_{0}+\tilde{Z}(s)\right) d s
$$

for $t \in[0, T]$. Inequality (3.81) provides a uniform bound on $\left|w_{\lambda_{n}}\right|_{C([0, T], E)}$, so by repeating the arguments for inequalities (3.37) and (3.38), it follows that

$$
\left|X_{\lambda_{n}}\right|_{C^{\beta}([0, T], V)} \leq c_{12}\left(1+\left\|X_{0}\right\|+|\tilde{Z}|_{C([0, T], E)}^{\rho}+|\tilde{Z}|_{C^{\beta}([0, T], V)}\right)
$$

for some $c_{12}>0$. Inequalities (3.80) and (3.81) verify inequality (3.79), so the sequence $\left(\exp \left[\rho_{\lambda_{n}}(Z)\right], n \in \mathbb{N}\right)$ is uniformly integrable and equality (3.73) is verified.

4. Some examples. The first example is a finite-dimensional stochastic equation with a nonlinear drift. Consider the equation

$$
d X(t)=f(X(t)) d t+\Phi d B(t),
$$

Copyright (C) by SIAM. Unauthorized reproduction of this article is prohibited. 
where $f: \mathbb{R}^{n} \rightarrow \mathbb{R}^{n}, \Phi \in \mathcal{L}\left(\mathbb{R}^{n}\right)$, and $(B(t), t \geq 0)$ is an $\mathbb{R}^{n}$-valued standard fractional Brownian motion with Hurst parameter $H \in(0,1)$. This case can be subsumed in the infinite-dimensional results given here, though some of the assumptions and the results simplify significantly. Let $E=V=\mathbb{R}^{n}, S(t)=I$ for $t \in \mathbb{R}_{+}$, and assume that $Q=\Phi \Phi^{*}$ is positive definite. The process

$$
\left(\int_{0}^{t} \Phi d B, t \in[0, T]\right)
$$

has sample paths in $C^{\beta}\left([0, T], \mathbb{R}^{n}\right)$ for $0<\beta<H$. If $f: \mathbb{R}^{n} \rightarrow \mathbb{R}^{n}$ is Borel measurable and

$$
\|f(x)\| \leq k_{1}(1+\|x\|)
$$

for some $k_{1}>0$ and all $x \in \mathbb{R}^{n}$, then for $H \in(0,1 / 2)$ there is one and only one weak solution of (4.1) by Theorem 3.3. If, additionally, it is assumed that

$$
\|f(x)-f(y)\| \leq k\|x-y\|^{\gamma}
$$

for all $x, y \in \mathbb{R}^{n}$ and some $\gamma>1-\frac{1}{2 H}$, then for $H \in(1 / 2,1)$ there is one and only one weak solution. In each of these cases, the probability measure of the solution is mutually absolutely continuous with respect to the probability measure of the process $(\Phi B(t), t \in[0, T])$.

Now, replace the inequality in (4.2) by

$$
\|f(x)\| \leq k_{1}\left(1+\|x\|^{\rho}\right)
$$

for some $\rho \geq 1$ and $k_{1}>0$. Assume that $f: \mathbb{R}^{n} \rightarrow \mathbb{R}^{n}$ is continuous and satisfies

$$
\langle f(x)-f(y), x-y\rangle \leq k_{3}\|x-y\|^{2}
$$

for some $k_{3}>0$ and all $x, y \in \mathbb{R}^{n}$. If $H \in(1 / 2,1)$, then assume that

$$
\|f(x)-f(y)\| \leq k_{4}\left(1+\|x\|^{q}+\|y\|^{q}\right)\|x-y\|^{\gamma}
$$

for some $q \geq 1, k_{4}>0, \gamma>1-\frac{1}{2 H}$. For $H \in\left(0, \frac{1}{2}\right)$ Theorem 3.6 can be used to verify that the probability law of the solution of (4.1) is mutually absolutely continuous with respect to the probability law of $(\Phi B(t), t \in[0, T])$. Furthermore, there is one and only one mild solution of (4.1); in fact, since the state space is finite-dimensional, the mild solution is a strong solution. For $H \in\left(\frac{1}{2}, 1\right)$ Theorem 3.7 can be used to verify mutual absolute continuity and one and only one mild solution as for the case $H \in\left(0, \frac{1}{2}\right)$. Note that inequalities (4.4)-(4.6) are satisfied for the important case of models where $f$ is a polynomial of odd degree with a negative leading coefficient.

The second example is a stochastic parabolic equation of $2 m$ th order:

$$
\frac{\partial u}{\partial t}(t, \xi)=\left[L_{2 m} u\right](t, \xi)+f(u(t, \xi))+\eta(t, \xi)
$$

for $(t, \xi) \in[0, T] \times \mathcal{O}$ with the initial condition

$$
u(0, \xi)=x(\xi)
$$

for $\xi \in \mathcal{O}$ and the Dirichlet boundary condition

$$
\frac{\partial^{k} u}{\partial v^{k}}(t, \xi)=0
$$

Copyright $@$ by SIAM. Unauthorized reproduction of this article is prohibited. 
for $(t, \xi) \in[0, T] \times \partial \mathcal{O}, k \in\{0, \ldots, m-1\}$, with $\frac{\partial}{\partial v}$ denoting the conormal derivative, $\mathcal{O}$ a bounded domain in $\mathbb{R}^{d}$ with a smooth boundary, and $L_{2 m}$ a $2 m$ th order uniformly elliptic operator

$$
L_{2 m}=\sum_{|\alpha| \leq 2 m} a_{\alpha}(\xi) D^{\alpha}
$$

with $a_{\alpha} \in C_{b}^{\infty}(\mathcal{O})$. For example, if $m=1$, then this equation is called the stochastic heat equation. The process $\eta$ denotes a space-dependent noise process that is fractional in time with the Hurst parameter $H \in(0,1)$ and, possibly, in space. The system (4.7)-(4.9) is modeled as

$$
\begin{aligned}
d X(t) & =A X(t) d t+F(X(t)) d t+\Phi d B(t), \\
X(0) & =x
\end{aligned}
$$

in the space $V=L^{2}(\mathcal{O})$, where $A=L_{2 m}$,

$$
\operatorname{Dom}(A)=\left\{\varphi \in H^{2 m}(\mathcal{O}) \mid \frac{\partial^{k}}{\partial v^{k}} \varphi=0 \text { on } \partial D \text { for } k \in\{0, \ldots, m-1\}\right\},
$$

$F: V \rightarrow V$ is the operator, $F(x)(\xi)=f(x(\xi)), x \in V, \xi \in \mathcal{O}, \Phi \in \mathcal{L}(V)$ defines the space correlation of the noise process, and $(B(t), t \geq 0)$ is a cylindrical standard fractional Brownian motion in $V$ (formally, $\eta(t, \cdot)=\Phi(\partial / \partial t) B(t, \cdot)$ ). For $\Phi=I$, the noise process is uncorrelated in space. It is well known that $A$ generates an analytic semigroup $(S(t), t \geq 0)$. Furthermore

$$
|S(t) \Phi|_{\mathcal{L}_{2}(V)} \leq|S(t)|_{\mathcal{L}_{2}(V)}|\Phi|_{\mathcal{L}(V)} \leq c t^{-\frac{d}{4 m}}
$$

for $t \in[0, T]$, so if

$$
H>\frac{d}{4 m}
$$

then the conditions of Proposition 2.6 are satisfied with $\gamma=\frac{d}{4 m}$. Therefore, for any $\Phi \in \mathcal{L}(V)$, the stochastic convolution process

$$
\left(\int_{0}^{t} S(t-r) \Phi d B(r), t \in[0, T]\right)
$$

is well-defined and has a version with $C^{\beta}([0, T], V)$ sample paths for $\beta \geq 0$ satisfying

$$
\beta<H-\frac{d}{4 m} \text {. }
$$

Note that the condition (4.13) extends the well-known result for a standard Wiener process $\left(H=\frac{1}{2}\right)$.

Theorems 3.3 and 3.4 are applied to the present example. Assume inequality (4.13) and let $\Phi$ be boundedly invertible on $V$. Furthermore, let $f: \mathbb{R} \rightarrow \mathbb{R}$ be measurable and satisfy

$$
|f(\xi)| \leq k_{1}(1+|\xi|), \quad \xi \in \mathbb{R} .
$$

Copyright (c) by SIAM. Unauthorized reproduction of this article is prohibited. 
By the preceding part of this example, conditions (H1)-(H3) are satisfied for $E=V=L^{2}(\mathcal{O})$ and the map $F: V \rightarrow V$ has at most linear growth. Thus by Theorem 3.3 if $H<\frac{1}{2}$, then there exists a unique weak solution to (4.11).

If $H>\frac{1}{2}$, some additional conditions are required. Assume that

$$
\frac{d}{4 m}<\frac{1}{2}
$$

(which is more restrictive than (4.13)) and suppose that

$$
|f(\xi)-f(\lambda)| \leq k|\xi-\lambda|^{\gamma}, \quad \xi, \lambda \in \mathbb{R},
$$

for some $k>0$ and $\gamma>0$,

$$
\frac{H-1 / 2}{H-d / 4 m}<\gamma \leq 1
$$

Then, letting $\beta$ be such that $\beta<H-\frac{d}{4 m}$ and $\gamma \beta>H-\frac{1}{2}$, it is clear that all of the conditions of Theorem 3.4 are verified so there is a unique weak solution to (4.11).

The third example is a one-dimensional stochastic equation of reaction-diffusion type. Consider the equation

$$
\frac{\partial u}{\partial t}(t, \xi)=\frac{\partial^{2} u}{\partial \xi^{2}}(t, \xi)+f(u(t, \xi))+\eta(t, \xi)
$$

for $(t, \xi) \in(0, T) \times(0,1)$ and

$$
\begin{aligned}
u(0, \xi) & =x_{0}(\xi), \\
\frac{\partial u}{\partial \xi}(t, 0) & =\frac{\partial u}{\partial \xi}(t, 1)=0
\end{aligned}
$$

for $(t, \xi) \in(0, T) \times(0,1)$, where $f$ and $\eta$ are given in the previous example (with $\mathcal{O}=(0,1))$. The above formal equation can be rewritten in the form (4.11) with $V=L^{2}([0,1]), A=\frac{\partial^{2}}{\partial \xi^{2}}$,

$$
\operatorname{Dom}(A)=\left\{\phi \in H^{2}([0,1]): \frac{\partial}{\partial \xi} \phi(0)=\frac{\partial}{\partial \xi} \phi(1)=0\right\},
$$

where $\Phi \in \mathcal{L}(V)$ and $F$ is as given in the preceding example. The semigroup generated by $A$ satisfies the estimate (4.12) (with $m=d=1$ ), so if $f$ satisfies the conditions of the previous example, the same conclusions on existence and uniqueness of the weak solution are obtained.

However, it is desirable to relax condition (4.15) of the linear growth of the function $f$, which is very restrictive in view of reaction-diffusion models, where $f$ is often a polynomial. Let $H>1 / 2$ and assume that

$$
\begin{gathered}
|f(\xi)| \leq k\left(1+|\xi|^{\rho}\right), \\
(f(\xi)-f(\lambda)) \operatorname{sgn}(\xi-\lambda) \leq k(\xi-\lambda), \\
|f(\xi)-f(\lambda)| \leq k\left(1+|\xi|^{q}+|\lambda|^{q}|\xi-\lambda|^{\gamma}\right.
\end{gathered}
$$

Copyright $@$ by SIAM. Unauthorized reproduction of this article is prohibited. 
for all $\xi, \lambda \in \mathbb{R}$ and some universal constants $\rho>0, q>0, k>0$ and $\gamma$ satisfying

$$
\frac{H-1 / 2}{H-1 / 4}<\gamma \leq 1
$$

Note that these conditions are satisfied if $f$ is Lipschitz or if $f$ is a polynomial of odd degree with a negative leading coefficient.

The conditions of Theorem 3.7 are verified now. Take the state space $E=$ $C([0,1])$. It is well known that the restriction of $A$ to $E$ generates a strongly continuous semigroup of contractions on E. By Proposition 2.6 the stochastic convolution

$$
\left(\int_{0}^{t} S(t-r) \Phi d B(r), t \in[0, T]\right)
$$

has $C^{\beta}\left([0, T], V_{\delta}\right)$ sample paths for $\beta+\delta<H-1 / 4$, and, hence, by the Sobolev embedding theorem, in the space $C([0, T], E) \cap C^{\beta}([0, T], V)$ for $0<\beta<H-1 / 4$ (by (4.23) $\beta$ can be chosen such that $\beta \gamma>H-1 / 2$ ). It remains to verify the conditions imposed on $F$. The polynomial growth condition (3.41), the "dissipativity of $F-k I$ on $V$ " (3.66), and the local Hölder continuity of the form (3.67) follow easily from the corresponding conditions on $f$, that is, (4.20), (4.21), and (4.22). The dissipativity of $F-k I$ on $E$ (3.42) is a well-known consequence of (4.21) by the characterization of the subdifferential of the norm on $E=C([0,1])$ (cf. [32]). The characterization of the subdifferentials for this example is as follows: Given $x \in E$, let $M_{x}=\left\{\xi \in[0,1] ;|x(\xi)|=\|x\|_{E}\right\}$. Then $\mu \in \delta\|x\|_{E}$ if and only if the following three conditions are satisfied: (i) $\mu$ is a Radon measure on $[0,1]$ with $\|\mu\|=1$; (ii) the support of $\mu$ is contained in $M_{x}$; and (iii) $\int_{\Gamma} \operatorname{sgn} x(\xi) \mu(d \xi) \geq 0$ for each Borel set $\Gamma$ in $[0,1]$. In particular if $x \in E$ has the property that $M_{x}=\left\{\xi_{0}\right\}$, then $\delta\|x\|_{E}=\delta_{\xi_{0}}$ for $x\left(\xi_{0}\right)=\|x\|_{E}$ and $\delta\|x\|_{E}=-\delta_{\xi_{0}}$ for $x\left(\xi_{0}\right)=-\|x\|_{E}$, where $\delta_{\xi_{0}}$ is the Dirac distribution at $\xi_{0}$. The family of $x \in E$ with this latter property is dense in $E$. Therefore, all of the conditions of Theorem 3.7 are satisfied, and it follows that there is a unique weak solution in the present case.

\section{REFERENCES}

[1] E. Alòs, O. Mazet, And D. Nualart, Stochastic calculus with respect to Gaussian processes, Ann. Probab., 29 (2001), pp. 766-801.

[2] P. CaithameR, The stochastic wave equation driven by fractional Brownian noise and temporally correlated smooth noise, Stoch. Dyn., 5 (2005), pp. 45-64.

[3] R. H. CAmeron And W. T. Martin, Transformations of Wiener integrals under translations, Ann. of Math. (2), 2 (1944), pp. 386-396.

[4] G. Da Prato and J. ZabczyK, Stochastic Equations in Infinite Dimensions, Encyclopedia Math. Appl. 44, Cambridge University Press, Cambridge, UK, 1992.

[5] G. Da Prato and J. Zabczyk, Ergodicity for Infinite-Dimensional Systems, London Math. Soc. Lecture Note Ser. 229, Cambridge University Press, Cambridge, UK, 1996.

[6] L. Decreusefond And A. S. Üstünel, Stochastic analysis of the fractional Brownian motion, Potential Anal., 10 (1999), pp. 177-214.

[7] L. Denis, M. Erraoui, and Y. Ouknine, Existence and uniqueness for solutions of one dimensional SDE's driven by an additive fractional noise, Stoch. Stoch. Rep., 76 (2004), pp. 409-427.

[8] T. E. Duncan, Some stochastic semilinear equations in Hilbert space with fractional Brownian motion, in Optimal Control and Partial Differential Equations, J. Menaldi, E. Rofman, and A Sulem, eds., IOS Press, Amsterdam, 2000, pp. 241-247.

[9] T. E. Duncan, Some processes associated with a fractional Brownian motion, in Mathematics of Finance, Contemp. Math. 351, AMS, Providence, RI, 2004, pp. 93-101.

[10] T. E. Duncan, Y. Hu, And B. Pasik-Duncan, Stochastic calculus for fractional Brownian motion I. Theory, SIAM J. Control Optim., 38 (2000), pp. 582-612. 
[11] T. E. Duncan, J. Jakubowski, and B. Pasik-Duncan, Stochastic integration for fractional Brownian motion in a Hilbert space, Stoch. Dyn., 6 (2006), pp. 53-75.

[12] T. E. Duncan, B. Maslowski, and B. Pasik-Duncan, Stochastic equations in Hilbert space with a multiplicative fractional Gaussian noise, Stochastic Process. Appl., 115 (2005), pp. 1357-1383.

[13] T. E. Duncan, B. Pasik-Duncan, And B. Maslowski, Fractional Brownian motion and stochastic equations in Hilbert spaces, Stoch. Dyn., 2 (2002), pp. 225-250.

[14] I. V. Girsanov, On transforming a class of stochastic processes by absolutely continuous substitution of measures, Teor. Verojatnost. i Primenen., 5 (1960), pp. 314-330.

[15] W. Grecksch and V. V. Anh, A parabolic stochastic differential equation with fractional Brownian motion input, Statist. Probab. Lett., 41 (1999), pp. 337-346.

[16] Y. Hu, Heat equations with fractional white noise potentials, Appl. Math. Optim., 43 (2001), pp. 221-243.

[17] Y. Hu, Integral transformations and anticipative calculus for fractional Brownian motions, Mem. Amer. Math. Soc., 175 (2005).

[18] Y. Hu, B. ØKSENDAL, AND T. Zhang, General fractional multiparameter white noise theory and stochastic partial differential equations, Comm. Partial Differential Equations, 29 (2004), pp. $1-23$.

[19] I. Karatzas and S. E. Shreve, Brownian Motion and Stochastic Calculus, Grad. Texts in Math. 113, Springer-Verlag, New York, 1988.

[20] R. S. Liptser and A. N. Shiryaev, Statistics of Random Processes I: General Theory, 2nd ed., Springer-Verlag, Berlin, 2001.

[21] B. Maslowski And D. Nualart, Evolution equations driven by a fractional Brownian motion, J. Funct. Anal., 202 (2003), pp. 277-305.

[22] B. Maslowski AND B. SChMALFUSS, Random dynamical systems and stationary solutions of differential equations driven by the fractional Brownian motion, Stochastic Anal. Appl., 22 (2004), pp. 1577-1607.

[23] R. H. Martin, JR., A global existence theorem for autonomous differential equations in a Banach space, Proc. Amer. Math. Soc., 26 (1970), pp. 307-314.

[24] R. Mikulevicius and B. L. Rozovsky, Martingale paths for stochastic PDE's, in Stochastic Partial Differential Equations: Six Perspectives, Math. Survey Monogr. 64, AMS, Providence, RI, 1999, pp. 243-325.

[25] S. Moret AND D. NuAlart, Onsager-Machlup functional for the fractional Brownian motion, Probab. Theory Related Fields, 124 (2002), pp. 227-260.

[26] I. Norros, E. Valkeila, and J. Virtamo, An elementary approach to a Girsanov formula and other analytical results on fractional Brownian motions, Bernoulli, 5 (1999), pp. 571-587.

[27] D. Nualart and Y. Ouknine, Regularization of differential equations by fractional noises, Stoch. Process. Appl., 102, (2002) pp. 103-116.

[28] B. Pasik-Duncan, T. E. Duncan, AND B. Maslowski, Linear stochastic equations in a Hilbert space with a fractional Brownian motion, in Stochastic Processes, Optimization, and Control Theory: Applications in Financial Engineering, Queueing Networks, and Manufacturing Systems, H. Yan, G. Yin, and Q. Zhang, eds., Springer-Verlag, New York, 2006, pp. 201-221.

[29] A. PAZY, Semigroups of Linear Operators and Applications to Partial Differential Equations, Appl. Math. Sci. 44, Springer-Verlag, New York, 1983.

[30] V. PIPIRAS AND M. S. TAQQU, Integration questions related to fractional Brownian motion, Probab. Theory Related Fields, 118 (2000), pp. 251-291.

[31] S. G. Samko, A. A. Kilbas, And O. I. Marichev, Fractional Integrals and Derivatives, Gordon and Breach, Yverdon, 1993.

[32] E. Sinestrari, Accretive differential operators, Boll. Un. Mat. Ital. B (5), 13 (1976), pp. 19-31.

[33] S. Tindel, C. A. Tudor, And F. Viens, Stochastic evolution equations with fractional Brownian motion, Probab. Theory Related Fields, 127 (2003), pp. 186-204.

Copyright $@$ by SIAM. Unauthorized reproduction of this article is prohibited. 